\title{
Sequencing dual-spreader crane operations: Mathematical formulation and heuristic algorithm
}

\author{
Shabnam Lashkari ${ }^{\mathrm{a}}$, Yong $\mathrm{Wu}^{\mathrm{b}}$, Matthew E. H. Petering ${ }^{\mathrm{a}, *}$ \\ ${ }^{a}$ Department of Industrial and Manufacturing Engineering, University of \\ Wisconsin-Milwaukee, P.O. Box 784, Milwaukee, WI 53201, USA \\ ${ }^{b}$ Department of International Business and Asian Studies, Griffith University, Gold \\ Coast Campus, QLD 4222 Australia
}

\begin{abstract}
This paper investigates the problem of scheduling a dual-spreader crane when lifts are subject to a weight limit. A mathematical model is formulated and a fast method for computing a lower bound on the optimal value is proposed. An efficient heuristic approach is designed and subsequently built into a simulated annealing framework to solve the problem. The optimization and heuristic approaches are tested on problem instances of various sizes. The results indicate that the optimization approach produces proven optimal solutions to small-sized instances but fails to solve instances of practical meaning. The heuristic approach can easily match the performance of the optimization approach for small instances and outperforms the optimization approach when tackling larger instances. On average, the heuristic approach produces solutions whose objective values are within $6 \%$ of the lower bound.
\end{abstract}

Keywords: dual-spreader crane, tandem-lift crane, integer programming, heuristics, planning and scheduling, container terminal, quay crane

\section{Introduction}

Today most overseas shipping of finished consumer goods is done via 20-, 40-, or 45-foot long steel containers aboard deep-sea container vessels. In addition, the amount of meat, fish, fruit, vegetables, and general foodstuffs

\footnotetext{
${ }^{*}$ Corresponding author. Tel: +1-414-229-3448, fax: +1-414-229-6958.

Email addresses: lashkari@uwm.edu (Shabnam Lashkari), yong.wu@griffith.edu.au (Yong Wu), mattpete@uwm.edu (Matthew E. H. Petering)

Preprint submitted to European Journal of Operational Research September 16, 2016
} 


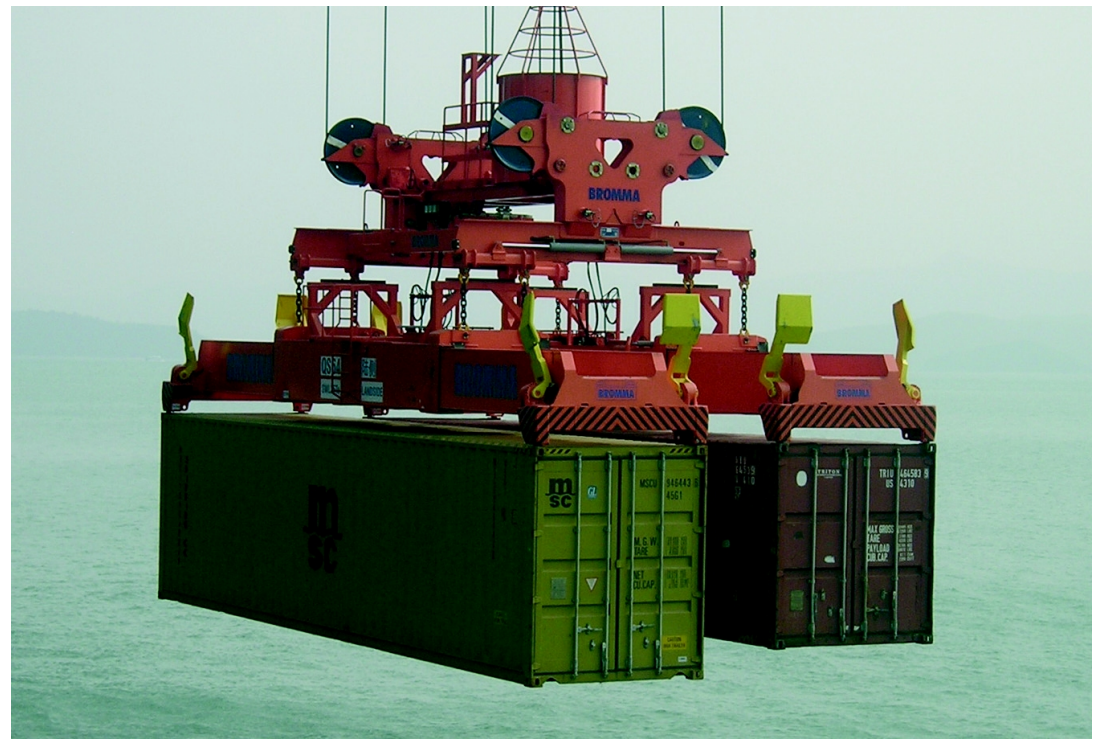

Figure 1: Double-spreader crane in operation (Source: http://www.portstrategy.com/ news101/port-operations/cargo-handling/multilift_for_and_against, accessed on 27 November 2015.)

shipped in refrigerated containers continues to increase. As the volume of freight shipped via steel shipping containers grows, it is becoming increasingly important to improve the operational efficiency of the port facilities where containerships are unloaded and loaded.

In this paper, we introduce a new mathematical problem that is inspired by the unloading of a containership. This problem is inspired by the recent development of a new kind of quay crane - a multi-spreader (i.e. tandem-lift) quay crane - that can lift more than one 40-foot container from a containership at the same time (Goussiatiner, 2007a,b). This new crane has an extra strong steel structure that allows heavier lifts to be performed. In contrast to traditional cranes, this new crane may deploy two or three spreaders (i.e. grappling devices) simultaneously, each of which can lift one 40-foot or two 20-foot containers (Goussiatiner, 2007a,b). Figure 1 shows a zoomed-in photo of a double-spreader quay crane in operation at a seaport container terminal.

With their superior specifications, tandem-lift quay cranes have the potential to significantly increase the productivity of seaport container terminals. However, due to a paucity of scheduling approaches for such cranes, 
this potential has not yet been fully realized. This motivates the current study. In this study we define a new mathematical problem that is inspired by the scheduling of a double-spreader (i.e. dual-spreader) quay crane. We call this new problem the dual-spreader crane scheduling problem (DSCSP).

We define the DSCSP as follows. Consider a set of containers (blocks, items) that are temporarily stored as inventory (e.g. on the deck of a ship). Due to space limitations, these containers are stacked directly on top of each other in a storage bay consisting of $S$ stacks and $T$ tiers. At time 0 , there are $E_{s}$ containers in stack $s$. The weight of the container in stack $s$, tier $t$ is given by $W_{s t}$. Consider the problem of sequencing the lifts made by one crane that will remove all containers from the bay. This crane can operate in two modes: single-spreader or dual-spreader mode. When in single-spreader mode, the crane may remove any single container from the top of any stack. This type of lift takes $H_{1}$ minutes. When in dual-spreader mode, the crane may simultaneously remove any two containers in the same tier from the top of any two adjacent stacks as long as the sum of their weights does not exceed $w_{\text {Limit }}$. This type of lift takes $\mathrm{H}_{2}$ minutes. Furthermore, the changeover (i.e. setup) time between modes is $C$ minutes. The crane can begin in either mode at time 0 with no initial setup cost. The goal is to sequence the individual lifts and changeovers of the crane so as to minimize the total time needed to remove all containers from the bay. To make the problem meaningful, we assume that $H_{1}<H_{2}<2 H_{1}$ and $\max \left\{W_{s t}\right\}<w_{\text {Limit }}<2 * \max \left\{W_{\text {st }}\right\}$.

Figure 2 shows an instance of the DSCSP. In this instance, $S=8, T=3$, $E_{s}=3$ for all $s$, and the weights $W_{s t}$ of all containers in the bay are shown in the upper-left corner of the figure. In addition, we assume that $w_{\text {Limit }}=10$, $H_{1}=1.5, H_{2}=1.8$, and $C=2.1$. Note that, even for this small instance, it is not easy to decide which containers should be lifted in single-spreader mode and which containers should be lifted in dual-spreader mode. Figure 2 shows a feasible crane lift sequence for this instance. This sequence consists of five dual-spreader lifts followed by four single-spreader lifts followed by five dual-spreader lifts. Two changeovers between spreader modes are required, so the total time needed to empty the bay - the makespan - is $10 \times 1.8+4 \times$ $1.5+2 \times 2.1=28.2$ minutes. We later show that this is not the optimal makespan for this instance.

This paper is organized as follows. Section 2 reviews the relevant literature. In Section 3, we present a mathematical formulation of the DSCSP and a fast method for computing a lower bound on the optimal objective value. In Section 4, we introduce a heuristic method that tries to find good solutions 


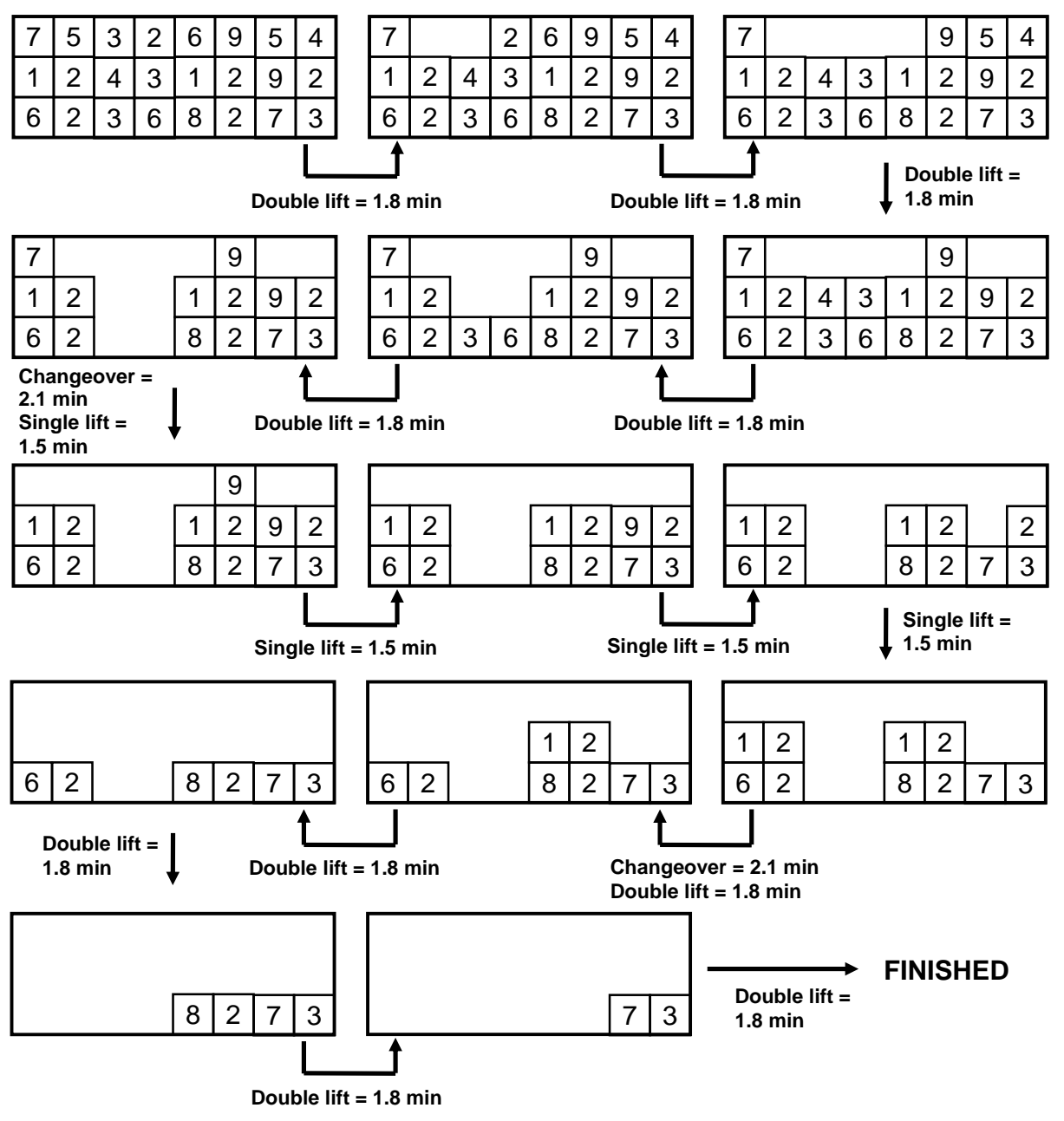

Figure 2 : Figure 1 . Feasible unloading sequence with.makespan 28.2 minutesfor a problem.instance of of size $3 \times 8$ with $w_{\text {Limit }}=10$.

to instances of the DSCSP within a short time. In Section 5, we describe the experimental setup and discuss the experimental results for two solution methods - standard integer programming and the heuristic method - on a set of 120 problem instances. We conclude this paper in Section 6.

\section{Literature Review}

The authors performed a rigorous search of the academic literature in order to find published works that previously addressed this topic. In par- 
ticular, the authors located all works published by one of the "big six" publishers - Elsevier, Springer, INFORMS, Taylor \& Francis, Wiley, and Palgrave Macmillan - that have a title containing any of the phrases "crane," "spreader," or "block relocation" in it. For works published by Elsevier and Taylor \& Francis, only items falling under the subject categories "decision sciences" and "economics, finance, business \& industry" respectively were considered. Industry journals were also searched. The results of the above literature search yielded several hundred articles, more than half of which concern the management of operations at seaport container transhipment terminals.

Ten articles surveying the literature on seaport container terminal operations were identified, including the works by Vis and De Koster (2003), Steenken et al. (2004), Stahlbock and Voß (2008), Bierwirth and Meisel (2010), Angeloudis and Bell (2011), Carlo et al. (2014a,b), Carlo et al. (2015), Gharehgozli et al. (2015b), and Bierwirth and Meisel (2015). Several of these articles mention that multi-spreader quay cranes (QCs) are an important new technology for container terminal operations. However, no article discusses a published paper that proposes a method for scheduling multi-spreader QCs.

Scores of papers consider the scheduling of cranes in industries unrelated to maritime shipping. For example, Dohn and Clausen (2010) solve a crane scheduling problem to optimize slab yard planning in a steel mill. Also, Kung et al. (2014) consider order scheduling of multiple stacker cranes on common rails in an automated storage and retrieval system. Peterson et al. (2014) propose a method for scheduling multiple factory cranes that operate on a common track.

Dozens of papers consider yard crane (YC) scheduling problems at seaport container terminals. For example, Cheung et al. (2002) propose methods for deploying YCs among storage blocks in a container terminal. At a more detailed level, Kim and Kim (1999) develop a math model and exact solution method for routing a single YC. Vis and Carlo (2010) develop a model for sequencing the operations of two automated stacking cranes (ASCs) in a container terminal. Li et al. (2012) propose a continuous time model for scheduling multiple YCs that can handle last minute job arrivals. Gharehgozli et al. (2015a) develop a math model and heuristic method for scheduling two ASCs in a single container storage block. Finally, Wu et al. (2015) present methods for scheduling multiple YCs that prevent YC interference and consider safety distance requirements.

Various methods have been developed for scheduling single-spreader QCs 
at seaport container terminals. For example, Imai et al. (2008) introduce a math model of the simultaneous berth and QC allocation problem and develop a genetic algorithm to find near-optimal solutions to the problem. Vacca et al. (2013), Türkoğulları et al. (2014), Iris et al. (2015), and Li et al. (2015) also consider variations of the simultaneous berth and QC allocation problem. Meisel and Bierwirth (2013) develop methods for solving the integrated berth allocation, QC allocation, and QC scheduling problem at seaport container terminals. Lee et al. (2011) and Chen et al. (2011) develop methods for scheduling QCs at indented berths. Kim and Park (2004) introduce a math model for scheduling QCs at a regular berth and develop exact and heuristic methods for solving problem instances. Moccia et al. (2006), Ng and Mak (2006), and Unsal and Oguz (2013) also propose various QC scheduling methods. Tang et al. (2014) consider a joint QC and truck scheduling problem. Also, Lee et al. (2015) develop an optimal algorithm for the general QC double-cycling problem.

Discussions of multi-spreader (i.e. tandem-lift) QCs are quite scarce in the literature. Chao and Lin (2011) present a methodology that trades off the various features of advanced QCs (including multi-spreader QCs) in order to choose a suitable advanced QC for any given container terminal. Xing et al. (2012) and Chen et al. (2014) develop methods for scheduling automated guided vehicles (AGVs) and yard trucks (YTs), respectively, when tandem-lift QCs are used at a container terminal. Choi et al. (2014) use a simulation methodology to develop an operating system that can increase the productivity of a container terminal where tandem-lift QCs are used. Several articles in industry journals - including those by McCarthy et al. (2007) and World Cargo News (2007) — contain general discussions of tandem-lift QCs but do not present results related to the scheduling or productivity of such cranes. On the other hand, Song (2011) discusses the productivity of tandem-lift QCs during real-life experiments conducted at Pusan Newport and proposes methods for conducting double cycling operations using such cranes. Finally, Goussiatiner $(2007 \mathrm{a}, \mathrm{b})$ generates plausible ship stowage configurations in order to compare the productivity of unloading such ships using single-spreader, dual-spreader, and triple-spreader QCs.

Overall, despite the existence of hundreds of published articles on industrial cranes, our literature search yielded no published model or method for scheduling multi-spreader cranes. In particular, we did not find any published method for deciding when a crane should switch between single-spreader and dual-spreader modes. Also, we did not find any method for deciding which 
containers in a storage bay should be lifted during single-spreader mode and dual-spreader mode. Thus, the problem considered in this article, and the methods for solving it, appear to be unique.

\section{Mathematical Model}

We now present a math model of the DSCSP. This is the better performing of two models that we developed for this problem. The other model can be found in the supplementary material accompanying this paper.

To facilitate the model development, we first convert the problem instance into a "binary array showing legal dual-spreader lifts" (BASLDSL). Figure 3 depicts the conversion of the instance in Figure 2 to $B A S L D S L$, where binary variables are used to indicate whether a dual-spreader lift could be performed on a pair of adjacent containers in the same tier. Without loss of generality, we use the left side of the pair to denote whether a "legal" dual-spreader lift can be performed within the given weight limit $w_{\text {Limit }}$. For example, the top-left ' 0 ' in BASLDSL indicates that the first and the second containers (from the left side) in the top tier cannot be dual-spreader lifted because their combined weight -12 - exceeds $w_{\text {Limit }}=10$. Also, the ' 1 ' adjacent to the topleft ' 0 ' indicates that the second and the third containers (from the left) in the top tier can be dual-spreader lifted because their combined weight - 8does not exceed $w_{\text {Limit }}$. In the original problem instance, we number the tiers $1, \ldots, T$ from bottom to top and the stacks $1, \ldots, S$ from left to right. In $B A S L D S L$, we use the terms tier $(1, \ldots, T$ from bottom to top) and column $(1, \ldots, S-1$ from left to right) to refer to various locations.

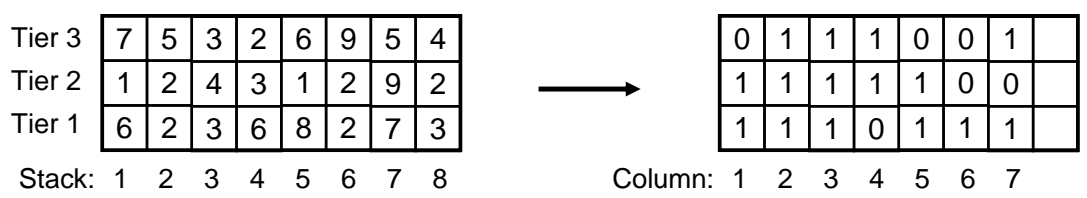

Figure 3: Cigure 3.renversipn of problem instance (left) inta binary array showing legal dual spreader lift (right) er lifts (right), assuming $w_{\text {Limit }}=10$.

\subsection{Mathematical formulation of the DSCSP}

Our mathematical model, model DSCSP, discretizes time into intervals. During each time interval, at most one (single-spreader or dual-spreader) lift may occur. The duration of an interval is therefore either $H_{1}$ or $H_{2}$ minutes 
depending on the type of operation performed. Between two consecutive intervals, at most one spreader changeover may occur (Figure 2).

The indices in model DSCSP are as follows:

$s \quad$ Stack, $s=1,2, \ldots, S$.

$t \quad$ Tier, $t=1,2, \ldots, T$.

$i \quad$ Time interval, $i=1,2, \ldots, I, I+1$

The input parameters in model DSCSP are as follows:

$S \quad$ Number of stacks in the storage bay. $S \geqslant 2$ to avoid triviality.

$T \quad$ Number of tiers in the storage bay.

$I \quad$ Number of time intervals available $(=S \times T$ to be conservative).

$E_{s} \quad$ Initial number of containers in stack $s, s=1, \ldots, S$.

$C$ Changeover time between single- and dual-spreader deployment.

$H_{1} \quad$ Handling time per lift using single spreader.

$\mathrm{H}_{2} \quad$ Handling time per lift using dual spreader.

$L_{s t}=1$ if the left side of the dual spreader can be used at stack $s$, tier $t$ in the original configuration $(s=1, \ldots, S-1, t=1, \ldots T)$ (binary). This parameter equals the value of the item in column $s$, tier $t$ of $B A S L D S L$.

The decision variables in model DSCSP are as follows:

$X_{s i}=1$ if a single-spreader lift is performed at the top of stack $s$ during time interval $i(s=1, \ldots, S, i=1, \ldots, I)$ (binary).

$Y_{s i}=1$ if a dual-spreader lift is performed in which the left (right) half of the spreader lifts the container that is on the top of stack $s(s+1)$ during time interval $i(s=1, \ldots, S-1, i=1, \ldots, I)$ (binary).

$G_{i}=1$ if a spreader changeover is made between time intervals $i-1$ and $i(i=2, \ldots, I)$ (binary).

$F_{i} \quad=1$ if we have finished removing all containers from the bay by the beginning of time interval $i(i=1, \ldots, I+1)$ (binary).

$N_{s i} \quad$ Number of containers in stack $s$ at beginning of time interval $i$ $(s=1, \ldots, S, i=1, \ldots, I+1)$ (integer, $\geqslant 0)$.

$R_{t i}=1$ if any containers are removed from tier $t$ during time interval $i(t=1, \ldots, T, i=1, \ldots, I)$ (binary). 
Objective function:

$$
\min M=\sum_{i=1}^{I}\left(\sum_{s=1}^{S} X_{s i} H_{1}+\sum_{s=1}^{S-1} Y_{s i} H_{2}\right)+\sum_{i=2}^{I} G_{i} C
$$

Subject to:

$$
\begin{aligned}
& \sum_{s=1}^{S} X_{s i}+\sum_{s=1}^{S-1} Y_{s i}+F_{i}=1 \quad i=1, \ldots, I \\
& \sum_{i=1}^{I+1} F_{i} \geqslant 1 \\
& \sum_{s=1}^{S-1} Y_{s, i-1}+G_{i}-1 \leqslant \sum_{s=1}^{S} X_{s i} \\
& \left.\begin{array}{rl}
\sum_{s=1}^{S} X_{s i}+\sum_{s=1}^{S-1} Y_{s, i-1}-1 & \leqslant G_{i} \\
G_{i}+\sum_{s=1}^{S} X_{s i}-1 & \leqslant \sum_{s=1}^{S-1} Y_{s, i-1}
\end{array}\right\} \\
& i=2, \ldots, I \\
& \sum_{s=1}^{S} X_{s, i-1}+G_{i}-1 \leqslant \sum_{s=1}^{S-1} Y_{s i} \\
& \left.\begin{array}{r}
\sum_{s=1}^{S-1} Y_{s i}+\sum_{s=1}^{S} X_{s, i-1}-1 \leqslant G_{i} \\
G_{i}+\sum_{s=1}^{S-1} Y_{s i}-1 \leqslant \sum_{s=1}^{S} X_{s, i-1}
\end{array}\right\} i=2, \ldots, I \\
& N_{s i} \leqslant T\left(1-F_{i}\right) \quad s=1, \ldots, S, i=1, \ldots, I+1 \\
& N_{s 1}=E_{s} \quad s=1, \ldots, S \\
& i=2, \ldots, I \\
& N_{1, i+1}=N_{1 i}-X_{1 i}-Y_{1 i} \quad i=1, \ldots, I \\
& N_{s, i+1}=N_{s i}-X_{s i}-Y_{s i}-Y_{s-1, i} \quad i=1, \ldots, I, s=2, \ldots, S-1 \\
& N_{S, i+1}=N_{S i}-X_{S i}-Y_{S-1, i} \quad i=1, \ldots, I
\end{aligned}
$$




$$
\begin{gathered}
\left.\begin{array}{c}
X_{1 i}+Y_{1 i} \leqslant N_{1 i} \quad i=1, \ldots, I \\
X_{s i}+Y_{s i}+Y_{s-1, i} \leqslant N_{s i} \quad i=1, \ldots, I, s=2, \ldots, S-1 \\
X_{S i}+Y_{S-1, i} \leqslant N_{S i} \quad \begin{array}{l}
i=1, \ldots, I \\
\sum_{t=1}^{T} R_{t i}=1 \quad i=1, \ldots, I
\end{array} \\
-T\left(1-X_{s i}\right) \leqslant \sum_{t=1}^{T} t R_{t i}-N_{s i} \leqslant T\left(1-X_{s i}\right) \quad i=1, \ldots, I, s=1, \ldots, S \quad(11) \\
-T\left(1-Y_{s i}\right) \leqslant \sum_{t=1}^{T} t R_{t i}-N_{s i} \leqslant T\left(1-Y_{s i}\right) \\
-T\left(1-Y_{s i}\right) \leqslant \sum_{t=1}^{T} t R_{t i}-N_{s+1, i} \leqslant T\left(1-Y_{s i}\right)
\end{array}\right\} \quad i=1, \ldots, I, s=1, \ldots, S-1 \\
Y_{s i}+R_{t i}-1 \leqslant L_{s t} s=1, \ldots, S-1, t=1, \ldots, T, i=1, \ldots, I
\end{gathered}
$$

The objective function (1) minimizes the makespan, $M$, which is the sum of the container handling and spreader changeover times. Constraint (2) ensures that at most one lift, either by the single spreader or dual spreader, can be performed during any time interval $i$; if no lift is made, then the "finished" binary variable $F_{i}$ should be set to 1 . Constraint (3) ensures that the process of removing containers from the bay is finished by the end of the last time interval. Constraint (4) forces a changeover to happen when switching from dual-spreader to single-spreader mode. This constraint has three expressions with the following structure: $A+B-1 \leqslant C ; C+A-1 \leqslant B$; and $B+C-1 \leqslant A$. These expressions ensure that if any two of the binary terms $A, B$, and $C$ equal 1 , then the third term equals 1 . Term $A$ indicates if a dual-spreader lift is made during time interval $i-1 ; B$ indicates if a spreader changeover is made between time intervals $i-1$ and $i$; and $C$ indicates if a single-spreader lift is made during time interval $i$. Constraint (5) is the same as (4) except that it considers the switch from single-spreader to dualspreader mode. 
Constraint (6) indicates that all stacks need to be empty before we can set $F_{i}$ to 1 . Constraint (7) initializes the stack heights for the first time interval. Constraint (8) updates the stack heights based on the lifts made during each time interval. Constraint (9) ensures that no lift is made from an empty stack. Constraint (10) ensures that container movement only happens in one tier during each time interval. Constraints (11) and (12) enforce the physical limitation that containers can only be picked up from the top tier. Constraints (10) and (12) together ensure that the dual spreader may only lift two containers that are in the same tier. Constraint (13) ensures that dual-spreader lifts do not violate the (weight-limit-respecting) binary values in $B A S L D S L$. The decision variable domains are included in the decision variable descriptions that precede the model.

\subsection{Lower bound computation}

The DSCSP is a challenging optimization problem. Although the NPhardness of this problem is still an open question, the results in Section 5 indicate that an optimization approach based on model DSCSP struggles to find proven optimal solutions to instances of modest size. In such cases, it is good to have a lower bound on the optimal objective value in order to estimate the quality of solutions produced by heuristic methods.

Algorithm 1 shows our approach for computing a lower bound on the optimal objective value for the DSCSP. It starts from the top tier and works downwards iteratively tier-by-tier (line 2). The consideration of each tier starts at its left end (line 3). Adjacent containers are checked in pairs to see whether they can be dual-spreader lifted (line 3). If so, the number of dual-spreader lifts is increased by one and these two containers are marked as handled (lines 5-6); otherwise, the number of single-spreader lifts is increased by one and only the left container of the pair is marked as handled (lines 78). The counting continues from left to right in the current tier until all containers in the tier are marked as handled, i.e. until the algorithm reaches the right side of the tier (line 4). Then the next tier is considered (line 2).

Once all containers in the storage bay are marked as handled, the total number of dual-spreader lifts is checked. If this number is not large enough to justify the cost of one spreader changeover, the algorithm assumes that only single-spreader lifts are performed and no changeovers are made (line 10). Otherwise it assumes that one changeover and the counted number of single-spreader and dual-spreader lifts are performed (line 12). 


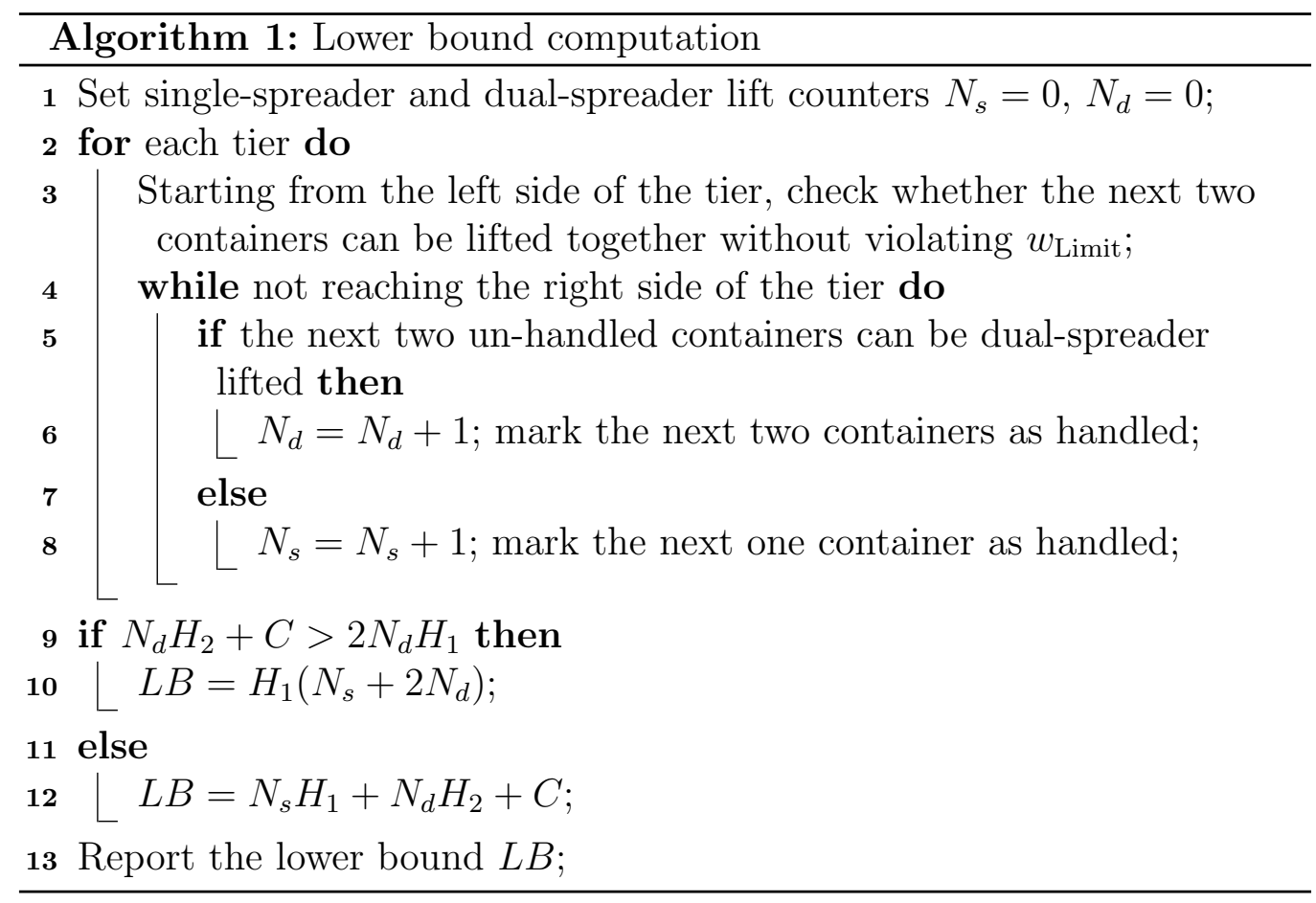

Theorem 1. Algorithm 1 computes a true lower bound on the optimal objective value for the DSCSP.

Proof 1. Note that a single changeover is only included in the lower bound computation if it is profitable; otherwise no changeover is included (lines 912). Thus, Algorithm 1 assumes the bare minimum number of changeovers. Consider the value of $N_{d}$ after the completion of the large "for" loop in Algorithm 1 (lines 2-8). We show that this value equals the maximum total number of dual-spreader lifts (i.e. dual lifts) that can be made. This fact, combined with the stipulation $\mathrm{H}_{2}<2 \mathrm{H}_{1}$, will prove the theorem.

Each dual lift is confined to a single tier. Thus, it suffices to show that the greedy method in Algorithm 1-which accepts all candidate dual lifts as soon as they appear during a left-to-right scan of a given tier-correctly computes the maximum number of dual lifts that can be made in any given tier.

We prove the correctness of the greedy method by induction. Let $D(n)$ be the maximum number of dual lifts that can be made solely on the rightmost $n$ containers in the tier at hand. Clearly, the greedy method correctly computes the maximum number of dual lifts that can be made on a set of 1 
or 2 containers in isolation; thus it correctly computes D(1) and D(2) when applied to those sets of containers in isolation.

To complete the proof, we need to show that, for $n \geqslant 3$, if the greedy method correctly computes $D(n-2)$ and $D(n-1)$, then it also correctly computes $D(n)$. There are two cases for the $n$ containers at hand. In case 1 , the two left-most containers cannot be feasibly dual lifted, i.e. they violate the weight limit. In this case, the greedy method skips over the left-most container; continues scanning at the second container; and computes $D(n-1)$ as the number of dual lifts made on the $n$ containers. But this is a correct computation for $D(n)$. Indeed, the left-most container is not eligible to participate in any dual lifts, so $D(n)=D(n-1)$ in this case. In case 2 , the two left-most containers can be feasibly dual lifted. In this case, the greedy method accepts the lift; skips over the two containers; continues scanning at the third container; and computes $1+D(n-2)$ as the number of dual lifts made on the $n$ containers. Any non-greedy method, by definition, would reject the dual lift; skip over the left-most container; continue scanning at the second container; and compute at most $D(n-1)$ as the number of dual lifts made on the $n$ containers. However, it is a general rule that $D(n-1) \leqslant D(n-2)+1$. Indeed, the addition of one container to the left-hand side of an existing row of $n-2$ containers increases the total number of dual lifts that can be performed on these containers by no more than one. This is because all dual lifts, except possibly the left-most such lift, found in the "optimal" set of dual lifts for the set of $n-1$ containers can be found in the set of $n-2$ containers. Thus, the dual lift tally computed by any non-greedy method cannot be more than that computed by the greedy method. Thus, the greedy method is "optimal" in case 2; it correctly computes $D(n)$ in this case. We have just proven the statement that begins this paragraph. Thus, the greedy method correctly computes $D(n)$ for all $n \geqslant 1$.

\section{Heuristic Approach}

Experimental results, discussed in Section 5, indicate that a direct math programming approach based upon model DSCSP is not a satisfactory solution method for large DSCSP instances with 50 or more containers. This motivated the authors to develop a heuristic method that can find good solutions to large problem instances within a reasonable time. We now describe this heuristic method. 
Our overall method consists of a constructive heuristic embedded within a simulated annealing (SA) metaheuristic. The constructive heuristic deterministically builds a feasible crane lift sequence based on the values of six parameters. During each iteration of the SA algorithm, the values of one or more parameters are changed to new, neighboring values, and a new feasible crane lift sequence is generated and evaluated.

We now provide a general description of the constructive heuristic, followed by a detailed description. Then we discuss the SA algorithm. The constructive heuristic is divided into two stages. In stage 1, the type of lift (single-spreader or dual-spreader) for each container is decided. In stage 2, a crane lift sequence - consisting of individual lifts and spreader changeoversis generated based on the output from stage 1 .

Our general approach to stage 1 is to iteratively accept or reject the dual-spreader lift opportunities that are shown in BASLDSL (right side of Figure 3). Note that the rejection of some dual-lift opportunities is often necessary to guarantee feasibility. For example, at least one of the dual-lift opportunities represented by two adjacent 'ones' in the same tier in $B A S L D$ $S L$ must be rejected; otherwise, the same container would be involved in two dual-spreader lifts - one with the container on its left, and one with the container on its right. Importantly, the dual-lift opportunities are not considered individually, but rather in batches of contiguous dual-lifts that are aligned vertically (i.e. in batches of consecutive 'ones' in the same column in $B A S L D S L)$. The consideration of such a batch often, but not always, results in all dual-lifts in the batch being accepted. To maintain feasibility, every acceptance is followed by the immediate rejection of all dual lift opportunities in the columns immediately to the right and left of the accepted batch's column in $B A S L D S L$. The output from stage 1 is a modified, or fixed, version of $B A S L D S L$ in which (1) the respective values are less than or equal to those in the initial $B A S L D S L$ and (2) there are no adjacent 'ones' in the same tier.

In stage 2, we use the fixed $B A S L D S L$ to label each container in the bay with a " $S$ " (" $D$ ") if it will be single-spreader (dual-spreader) lifted. Then we construct a feasible crane lift sequence by iteratively removing containers from the tops of the stacks in the bay if they match the current spreader being deployed. When no more lifts can be made using the current spreader, the spreader is changed. Lifting then continues using the new spreader. This process continues until no more containers remain in the bay. The makespan of the crane lift sequence is then computed.

We now describe the constructive heuristic in greater detail. Table 1 lists 
Table 1: Input parameters for the constructive heuristic

\begin{tabular}{|c|c|}
\hline InitialDirection & $\begin{array}{l}\text { Equals } 0 \text { (1) if heuristic initially moves down from } \\
\text { higher to lower tiers (up from lower to higher tiers). }\end{array}$ \\
\hline InitialTier & Tier at which heuristic begins $(=$ integer from 1 to $T$ ) \\
\hline ColumnCho & $\begin{array}{l}\text { The next value in this sequence of }[0,1) \text { real numbers } \\
\text { decides which column is considered next if two or more } \\
\text { columns } c \text { tie for having the greatest } \operatorname{Depth}(c) \text {. }\end{array}$ \\
\hline FixFullDepth YN [] & $\begin{array}{l}\text { The next value in this sequence of }[0,1) \text { real numbers } \\
\text { is compared to FullDepthChance to decide whether or } \\
\text { not to fix Depth }(c) \text { dual-spreader lifts in column } c \text {. }\end{array}$ \\
\hline ifts ToFix[ & $\begin{array}{l}\text { If FixFullDepth } Y N \text { indicates that fewer than Depth }(c) \\
\text { dual-spreader lifts in column } c \text { should be fixed, the } \\
\text { next value in this sequence of }[0,1) \text { real numbers de- } \\
\text { cides how many such lifts are fixed. }\end{array}$ \\
\hline InitialSpreader & $\begin{array}{l}\text { Forms a feasible crane lift sequence from the values in } \\
\text { the fixed } B A S L D S L \text {. Equals " } S \text { " " } D \text { ") if the lifting } \\
\text { begins in single-spreader (dual-spreader) mode. }\end{array}$ \\
\hline
\end{tabular}

the six parameters that provide input to this heuristic. Parameters 1 and 6 have two possible values; Parameter 2 has $T$ possible values; and Parameters 3,4 , and 5 are long sequences of $[0,1)$ real numbers. Parameters $1-5$ are used in stage 1 , and Parameter 6 is used in stage 2.

Algorithm 2 shows the pseudocode for stage 1 of the constructive heuristic. In this code, $A[*]$ indicates the first unused item in array $\mathrm{A}$. In stage 1 , $B A S L D S L$ is scanned in order to decide which potential dual-spreader lifts should and should not be performed. During this scan, the initial values in $B A S L D S L$ are gradually fixed to 1 or 0 , where a 1 (0) means that the associated dual-spreader lift will (will not) be performed. Parameter InitialTier in Table 1 specifies the starting point for this scan, which proceeds tier by tier (line 1). Parameter InitialDirection specifies whether the scan initially proceeds down from InitialTier to lower tiers or up from InitialTier to higher tiers (line 1$)$. We let $B A[c, t]$ denote the value in column $c$ and tier $t$ in $B A S L D S L$ (line 2).

During the consideration of tier Tier in $B A S L D S L$, the heuristic identifies the columns $c$ for which $B A[c$, Tier $]=1$ and is not already fixed (line 6 ). The value $\operatorname{Depth}(c)$ is then computed for each such column $c$. Depth $(c)$ equals 


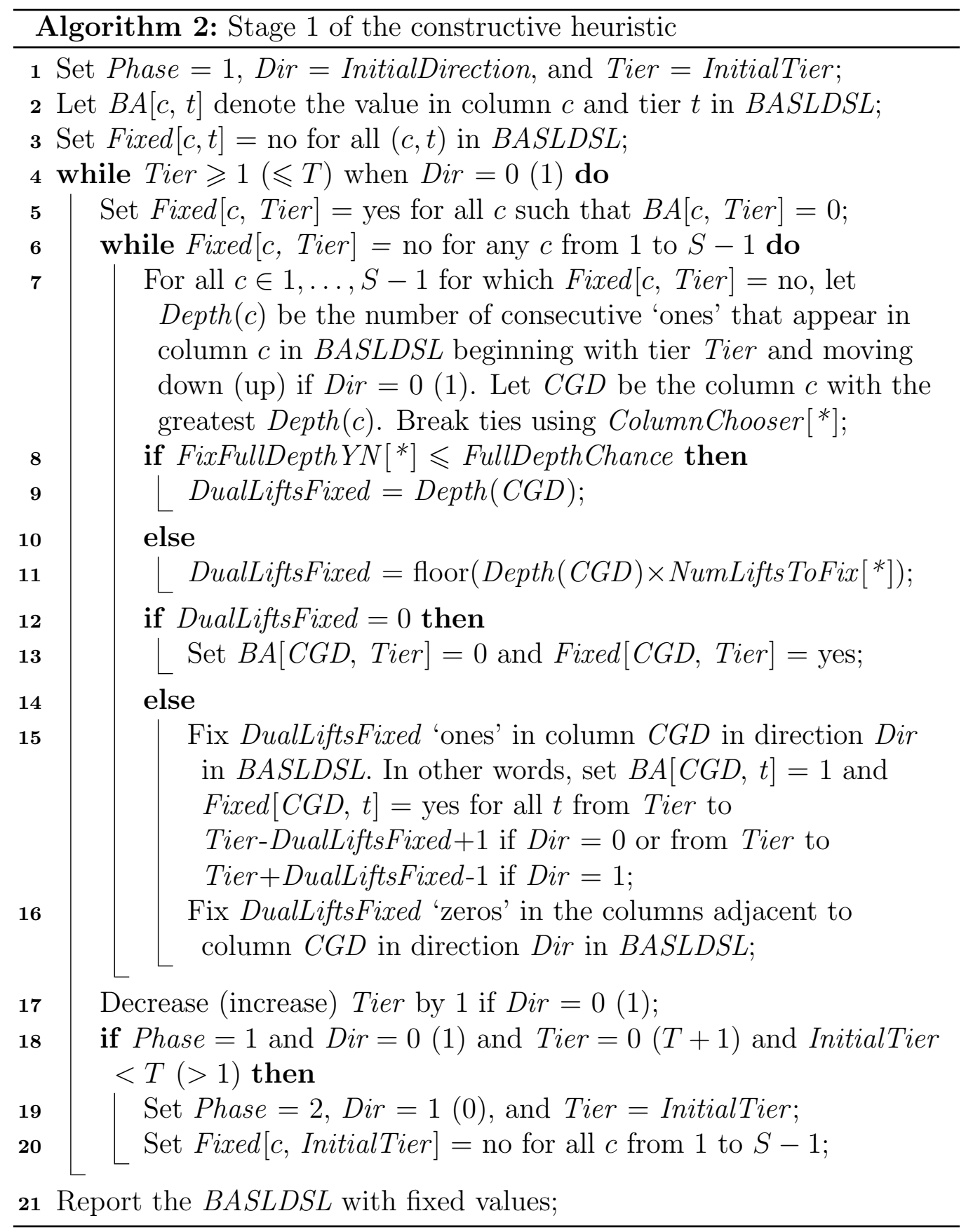




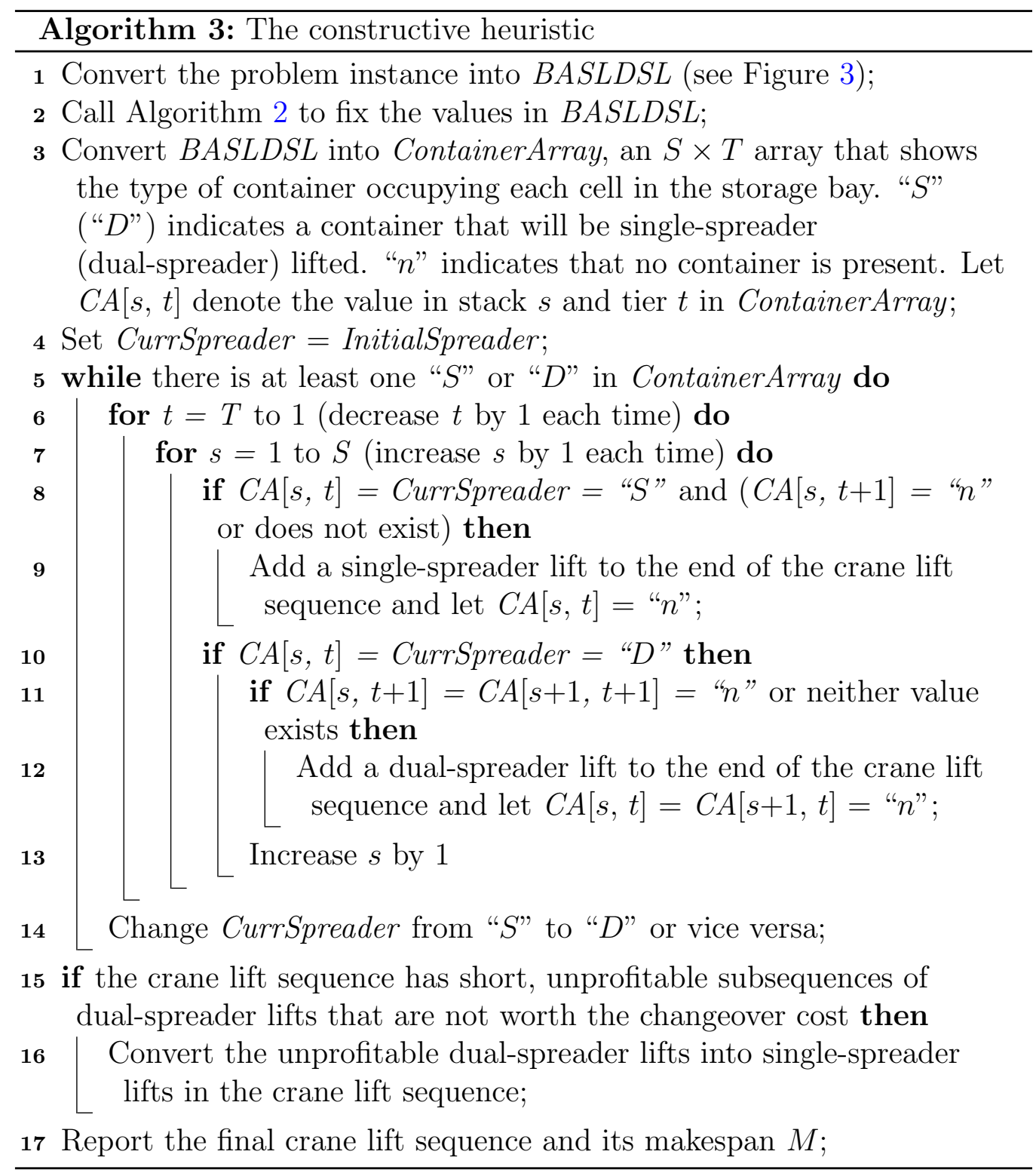

the number of consecutive 'ones' in column $c$ that begin at tier Tier and proceed upwards or downwards depending on the current scanning direction Dir (line 7). The dual-spreader opportunities in the column $c$ with the greatest Depth $(c)$ are the first candidates for acceptance. When two or more columns $c$ tie for having the greatest $\operatorname{Depth}(c)$, the next $[0,1)$ real number in array 
ColumnChooser [ ] breaks the tie and selects the "column with the greatest depth" (i.e. CGD) (line 7). In particular, if $L$ columns are tied, then the $n$th such column is selected if and only if $(n-1) / L \leqslant$ ColumnChooser $\left.{ }^{*}\right]<n / L$. The next $[0,1)$ real number in array FixFullDepth $Y N[]$ is then compared to global parameter FullDepthChance (line 8) to decide if all Depth(CGD) dual-spreader lift opportunities in column $C G D$ are accepted (line 9) or not (lines 10-11). If not, the next [0,1) real number in array NumLifts ToFix [ ] indicates what fraction of the dual-spreader lift opportunities are accepted, i.e. to what depth the 'ones' in the column are fixed. In particular, the number of 'ones' that are fixed equals floor $\left(\operatorname{Depth}(C G D) \times N u m L i f t s\right.$ ToFix $\left[{ }^{*}\right]$ ) (line 11). If this equals 0 (line 12), the single dual-lift opportunity in column $C G D$, tier Tier is rejected and the corresponding cell in BASLDSL is fixed to 0 (line 13). Otherwise, one or more dual-lift opportunities in column $C G D$ are accepted (i.e. a sub-column of 'ones' in BASLDSL is fixed) (line 15), and the values in all cells on either side of this sub-column are fixed to 0 (line 16). The latter step ensures that two adjacent 'ones' in the same tier in $B A S L D$ $S L$ are never both fixed. This concludes the handling of column $C G D$ in the current tier. Other columns $c$ are then considered one-at-a-time according to the ranking of their Depth $(c)$ values, and the above process repeats until all values in the current tier in $B A S L D S L$ have been fixed to 1 or 0 (lines 6-16).

The procedure then continues to the next tier in the current scanning direction Dir (line 17). Eventually all tiers in direction Dir will be scanned. At that point, the second phase of the scanning commences: the procedure jumps back to tier InitialTier, un-fixes all values in that tier, and begins scanning in the direction opposite from InitialDirection (lines 19-20). This second phase is undertaken if and only if InitialTier is a middle tier (line 18). After completion of the second phase, all tiers in $B A S L D S L$ have been scanned and all cells in $B A S L D S L$ have been fixed (line 21).

The overall constructive heuristic is shown in Algorithm 3. This heuristic first calls Algorithm 2 to fix the values in $B A S L D S L$ (line 2). It then converts the fixed $B A S L D S L$ into a ContainerArray, an $S \times T$ array that shows which containers in the storage bay will be single-spreader ( $S$ ") and dual-spreader ("D") lifted. A detailed crane lift sequence that starts using spreader InitialSpreader is then constructed in a straightforward manner using the values in ContainerArray (lines 4-14). If the end (middle) of the sequence contains short, unprofitable subsequences of dual-spreader lifts that are not worth the cost of one (two) spreader changeover(s), the unprofitable dual-spreader lifts are converted into single-spreader lifts (lines 15-16). Then the makespan of 


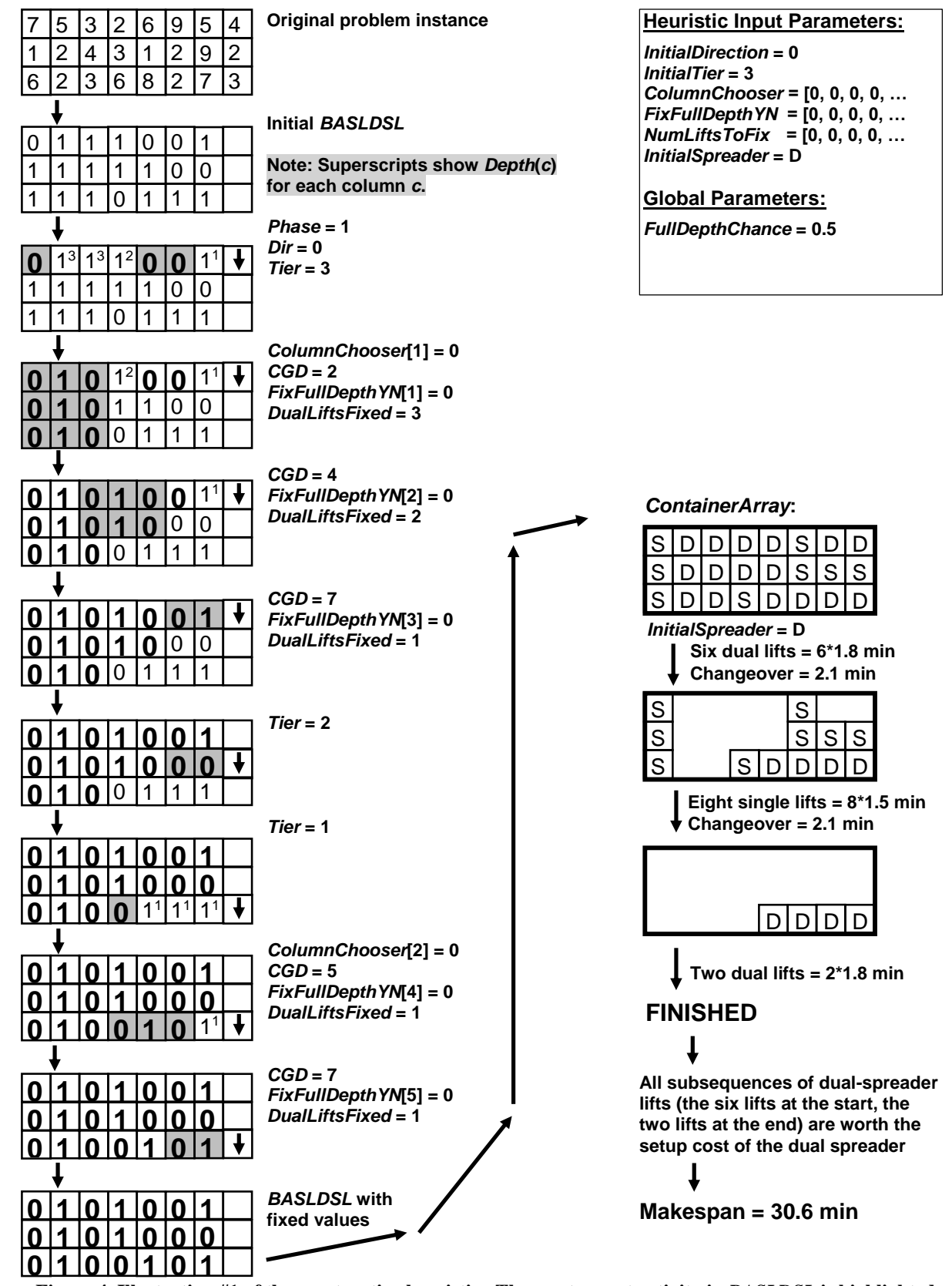

Figure 4. Illustration \#1 of the constructive heuristic. The most recent activity in BASLDSL is highlighted. Figure 4: Illustration \#1 of the constructive heuristic. The most recent activity in $B A S L D$ $S L$ is highlighted. Fixed values in $B A S L D S L$ are displayed in bold. 


\begin{tabular}{|c|c|c|c|c|c|c|c|c|}
\hline 7 & 5 & 3 & 2 & 6 & 9 & 5 & 4 & \multirow[t]{3}{*}{ Original problem instance } \\
\hline 1 & 2 & 4 & 3 & 1 & 2 & 9 & 2 & \\
\hline 6 & 2 & 3 & 6 & 8 & \begin{tabular}{|l|l}
2 & \\
\end{tabular} & 7 & 3 & \\
\hline \multicolumn{8}{|c|}{$\downarrow$} & \multirow{4}{*}{$\begin{array}{l}\text { Note: Superscripts show Depth(c } \\
\text { for each column c. }\end{array}$} \\
\hline 0 & 1 & 1 & 1 & 0 & \begin{tabular}{l|l}
0 & \\
\end{tabular} & 1 & & \\
\hline 1 & 1 & 1 & 1 & 1 & 0 & 0 & & \\
\hline 1 & 1 & 1 & 0 & 1 & 1 & 1 & & \\
\hline \multicolumn{8}{|c|}{$\downarrow$} & \multirow{4}{*}{$\begin{array}{l}\text { Phase }=1 \\
\text { Dir }=1 \\
\text { Tier }=2\end{array}$} \\
\hline 0 & 1 & 1 & 1 & 0 & $0 \mid$ & 1 & & \\
\hline $1^{1}$ & $1^{2}$ & $1^{2}$ & $1^{2}$ & $1^{1}$ & 0 & 0 & $\mathbf{t}$ & \\
\hline 11 & 1 & 1 & 0 & 1 & 11 & 1 & & \\
\hline \multicolumn{8}{|c|}{$\downarrow$} & \multirow{4}{*}{$\begin{array}{l}\text { ColumnChooser }[1]=.4 \\
\text { CGD }=3 \\
\text { FixFullDepthYM[1] }=.7 \\
\text { NumLiftsToFix[1] }=.6 \\
\text { DualLiftsFixed }=\text { floor }\left(2^{\star} .6\right)=1\end{array}$} \\
\hline 0 & 1 & 1 & 1 & 0 & 0 & 1 & & \\
\hline $1^{1}$ & 0 & 1 & 0 & $1^{1}$ & 0 & 0 & $\uparrow$ & \\
\hline 1 & 1 & 1 & 0 & 1 & 1 & 1 & & \\
\hline \multicolumn{8}{|c|}{$\downarrow$} & \multirow{4}{*}{$\begin{array}{l}\text { ColumnChooser }[2]=.8 \\
\text { CGD }=5 \\
\text { FixFullDepth } Y M[2]=0 \\
\text { DualLiftsFixed }=1\end{array}$} \\
\hline 0 & 1 & 1 & 1 & 0 & 0 & 1 & & \\
\hline $1^{1}$ & 0 & 1 & 0 & 1 & 0 & 0 & $\mathbf{T}$ & \\
\hline 1 & 1 & 1 & 0 & 1 & 1 & 1 & & \\
\hline \multicolumn{8}{|c|}{$\downarrow$} & \multirow{4}{*}{$\begin{array}{l}\text { CGD }=1 \\
\text { FixFullDepthYM[3] = } \\
\text { DualLiftsFixed }=1\end{array}$} \\
\hline 0 & 1 & 1 & 1 & 0 & 0 & 1 & & \\
\hline 1 & 0 & 1 & 0 & 1 & 0 & 0 & $\mathbf{1}$ & \\
\hline 1 & 1 & 1 & \begin{tabular}{|l|}
0 \\
\end{tabular} & 1 & 1 & 1 & & \\
\hline \multicolumn{8}{|c|}{$\downarrow$} & \multirow{4}{*}{ Tier $=3$} \\
\hline 0 & $1^{11}$ & $1^{1}$ & $1^{11}$ & 0 & 0 & $1^{1}$ & $\mathbf{t}$ & \\
\hline 1 & 0 & 1 & 0 & 1 & 0 & 0 & & \\
\hline 1 & 1 & 1 & 0 & 1 & 1 & 1 & & \\
\hline \multicolumn{8}{|c|}{$\downarrow$} & \multirow{4}{*}{$\begin{array}{l}\text { ColumnChooser }[3]=.9 \\
\text { CGD }=7 \\
\text { FixFullDepthYM[4] }=.9 \\
\text { NumLiftsToFix }[2]=.9 \\
\text { DualLiftsFixed }=\text { floor }\left(1^{\star} .9\right)=0\end{array}$} \\
\hline 0 & $1^{1}$ & $1^{1}$ & $1^{11}$ & 0 & 0 & 0 & $\boldsymbol{t}$ & \\
\hline 1 & 0 & 1 & 0 & 1 & 0 & 0 & & \\
\hline 1 & 1 & 1 & 0 & 1 & 1 & 1 & & \\
\hline \multicolumn{8}{|c|}{$\downarrow$} & \multirow{4}{*}{$\begin{array}{l}\text { ColumnChooser }[4]=.7 \\
C G D=4 \\
\text { FixFullDepth } Y M[5]=0 \\
\text { DualLiftsFixed }=1\end{array}$} \\
\hline 0 & $1^{11}$ & 0 & 1 & 0 & 0 & 0 & $\mathbf{t}$ & \\
\hline 1 & 0 & 1 & 0 & 1 & 0 & 0 & & \\
\hline \begin{tabular}{|l|}
1 \\
\end{tabular} & 1 & 1 & 0 & 1 & 1 & 1 & & \\
\hline \multicolumn{8}{|c|}{$\downarrow$} & \multirow{4}{*}{$\begin{array}{l}\text { CGD }=2 \\
\text { FixFullDepthYM6] }=0 \\
\text { DualLiftsFixed }=1\end{array}$} \\
\hline 0 & 1 & \begin{tabular}{l|l}
0 \\
\end{tabular} & 1 & 0 & 0 & 0 & 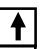 & \\
\hline 1 & 0 & 1 & 0 & 1 & 0 & 0 & & \\
\hline 1 & 1 & 1 & 0 & 1 & 1 & 1 & & \\
\hline & $\downarrow$ & & & & & & & Tier $=4$ \\
\hline 0 & 1 & 0 & 1 & 0 & & 0 & & $\begin{array}{l}\text { Phase }=2 \\
\text { Dir }=0\end{array}$ \\
\hline $1^{2}$ & 0 & $1^{2}$ & 0 & $1^{2}$ & 0 & 0 & $\nabla$ & Tier $=2$ \\
\hline
\end{tabular}
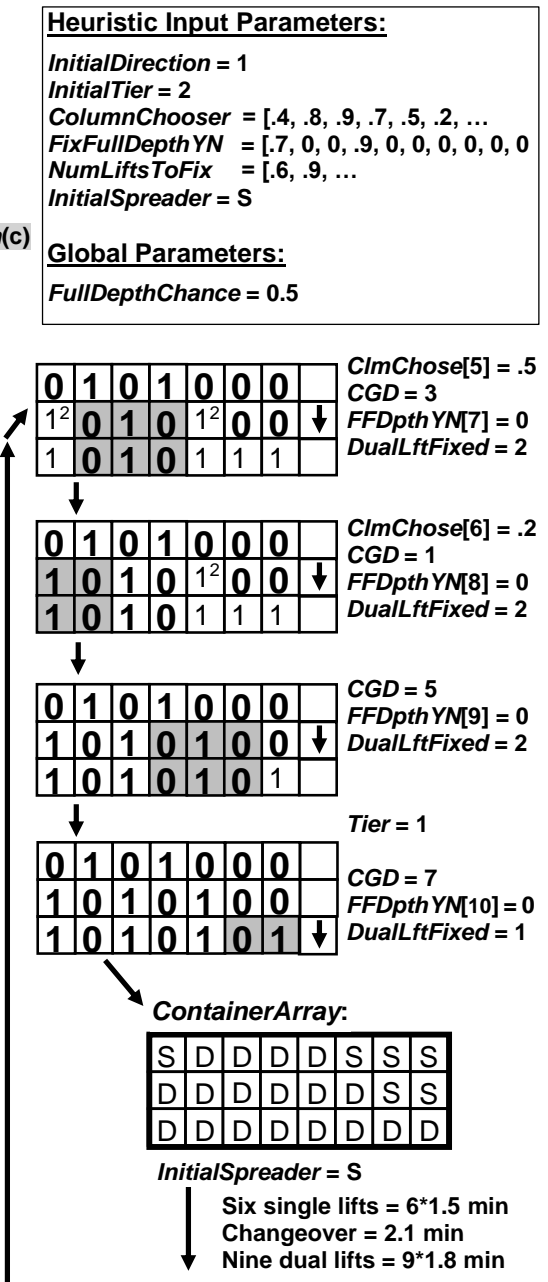

FINISHED

$$
\downarrow
$$

All subsequences of dual-spreader lifts are worth the setup cost of the dual spreader

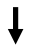

Makespan $=27.3 \mathrm{~min}$

Figure 5. Illustration \#2 of the constructive heuristic. The most recent activity in BASLDSL is highlighted. Figure 5: Illustration \#2 of the constructive heuristic. The most recent activity in $B A S L D$ $S L$ is highlighted. Fixed values in $B A S L D S L$ are displayed in bold. 


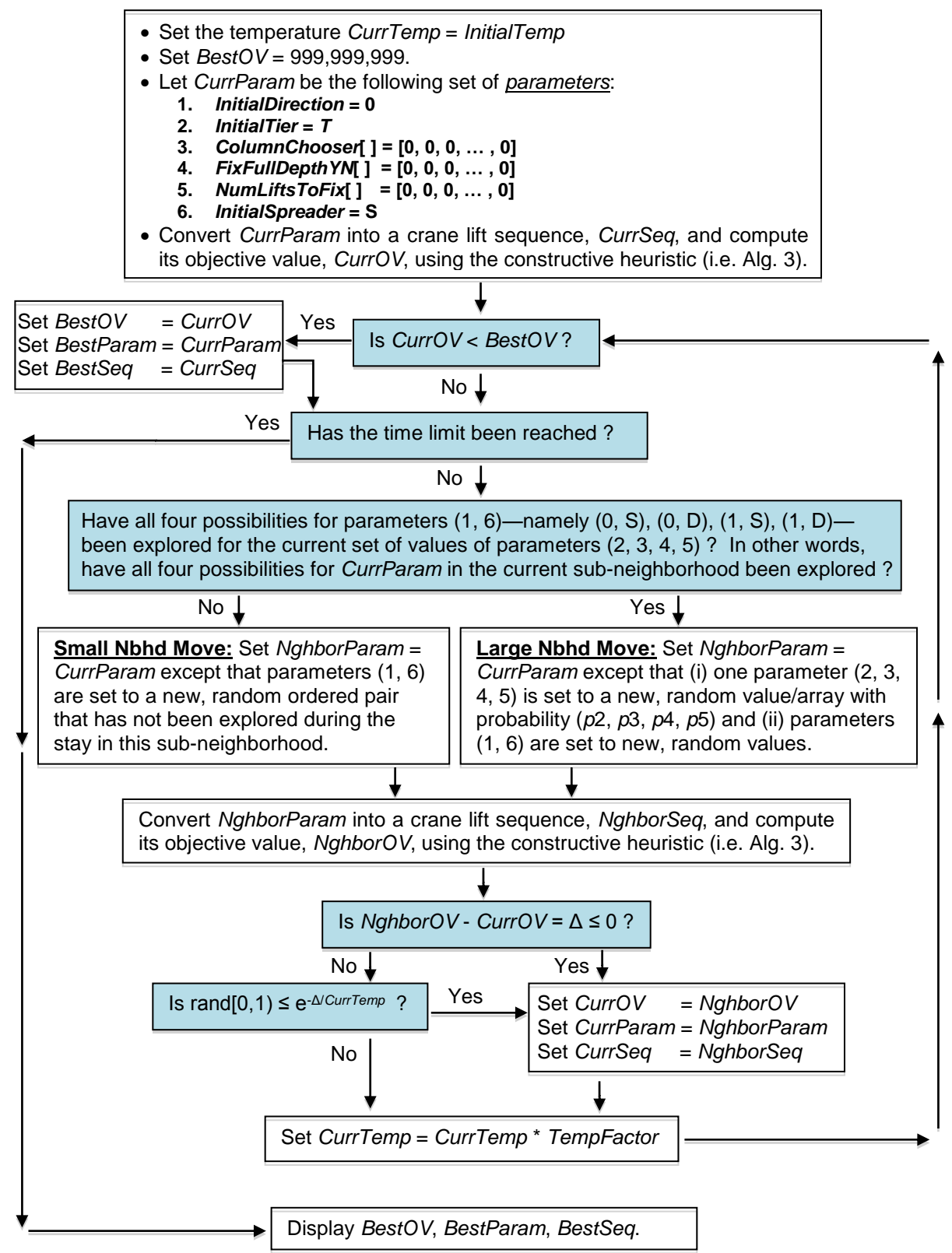

Figure 6: Overall logic of the simulated annealing metaheuristic.

Figure 6. Overall logic of the simulated annealing metaheuristic. 
the resulting crane lift sequence is computed (line 17).

Figures 4 and 5 show how the constructive heuristic generates two different crane lift sequences for the problem instance shown in Figure 3 based on two sets of input parameters. In each figure, the original problem instance and its conversion into the initial, tentative $B A S L D S L$ are shown in the upper-left. The values of the six input parameters are shown in the upper-right. The left side of each figure shows how Algorithm 2 gradually fixes the values in $B A S L D S L$ using the first five parameters. Note that only the first several values in arrays ColumnChooser [ ], FixFullDepthYN [ ], and NumLiftsToFix[ ] are utilized; the other values are not used. The conversion of the fixed BASLDSL into ContainerArray; the generation of a detailed crane lift sequence; and the makespan computation are shown in the bottomright. Notice that the fixed BASLDSL, ContainerArray, and makespan are quite different in the two figures. Indeed, the constructive heuristic is able to generate vastly different crane lift sequences when the input parameters are changed. It turns out that the makespan shown in Figure 5 is optimal.

Figure 6 shows how the constructive heuristic is embedded within a simulated annealing (SA) framework. The SA procedure begins by initializing the six input parameters, collectively referred to as CurrParam, and converting them into a feasible crane lift sequence CurrSeq and makespan CurrOV using the constructive heuristic. In each SA iteration, CurrParam is used to generate a new set of parameter values NghborParam; the constructive heuristic converts NghborParam into a feasible crane lift sequence; and the laws of simulated annealing decide if $N$ ghborParam replaces CurrParam.

Two kinds of neighborhood moves-small moves and large moves - are utilized. In a small move, a new, random combination of values for parameters 1 and 6-InitialDirection and InitialSpreader - is considered and the other parameters remain unchanged. In a large move, a new, random value for parameter $(2,3,4,5)$ is considered with probability $(p 2, p 3, p 4, p 5)$ and a new, random combination of values for parameters 1 and 6 is considered $(p 2+p 3+p 4+p 5=1)$. A small move is made whenever it can produce a new set of parameter values that has not yet been explored. Otherwise, a large move is initiated. When a predefined time limit is reached, the SA procedure terminates and the best crane lift sequence that was found is displayed. 


\section{Experimental Setup, Results and Discussion}

The heuristic method from Section 4 and model DSCSP from Section 3 were coded into MS Visual C++ 2010 Professional. IBM ILOG Concert Technology was used to define model DSCSP within $\mathrm{C}++$ and call the MILP solver IBM ILOG CPLEX 12.5 to solve instances defined in text files. To avoid running out of memory, the CPLEX "node file storage parameter" is set to 3. That is, the information for every unexplored node in the branchand-cut tree is stored on the hard disk and compressed. Otherwise, default CPLEX settings are used. All results are obtained using a desktop computer with the Windows 7 Enterprise 64-bit operating system, an Intel Core i7-4770 processor with eight $3.4 \mathrm{GHz}$ cores, and $16 \mathrm{~GB}$ of RAM.

We consider a total of 120 problem instances - 30 instances for each of the problem sizes $3 \times 8,5 \times 10,10 \times 23$, and $50 \times 50$. A problem of size $T \times S$ has $S$ stacks, $T$ tiers, and $T$ containers in stack $s$ for all $s$ at time 0 . In all instances, we assume that the container weights $W_{s t}$ take integer values from 1 to 9 . We also assume that $w_{\text {Limit }}=10, H_{1}=1.5, H_{2}=1.8$, and $C=2.1$. Among the 30 instances for each problem size, $(10,10,10)$ instances have (light, medium, heavy) container weights. In the medium instances, the weight of each container follows a discrete uniform distribution over the values $\{1,2$, $3,4,5,6,7,8,9\}$. In the light instances, the weight of each container has a $\{15 \%, 15 \%, 15 \%, 15 \%, 20 \%, 5 \%, 5 \%, 5 \%, 5 \%\}$ chance of taking the value $\{1,2,3,4,5,6,7,8,9\}$. In the heavy instances, the weight of each container has a $\{5 \%, 5 \%, 5 \%, 5 \%, 20 \%, 15 \%, 15 \%, 15 \%, 15 \%\}$ chance of taking the value $\{1,2,3,4,5,6,7,8,9\}$. Text files defining all problem instances can be found in the supplementary material accompanying this paper.

Table 2 shows the settings used in the heuristic algorithm for each of the four problem sizes. Preliminary experiments were performed to determine these settings. The results of these preliminary experiments, not shown here, indicated that performance was most consistent when the neighbor probabilities $\left(p_{2}, p_{3}, p_{4}, p_{5}\right)$ equal $(.25, .25, .25, .25)$ (Figure 6$)$. We also found that performance improves with a higher FullDepthChance (line 8 of Algorithm 2, middle of Table 1) as the problem size increases. We hypothesize that this is because instances with more tiers can have larger batches of dual lift opportunities that are aligned vertically (i.e. longer strings of consecutive 'ones' in the same column in the initial $B A S L D S L$ ). As the size of such a batch increases, it may be increasingly important to accept all dual lift opportunities in the batch to prevent "disrupting" the batch with a single rejected dual 
Table 2: Parameter settings for the heuristic method

\begin{tabular}{lcccc}
\hline Problem Size & $3 \times 8$ & $5 \times 10$ & $10 \times 23$ & $50 \times 50$ \\
\hline Computational time limit (seconds) & 10 & 60 & 600 & 600 \\
FullDepthChance & 0.5 & 0.7 & 0.8 & 0.99 \\
Neighbor probabilities $\left(p_{2}, p_{3}, p_{4}, p_{5}\right)$ & \multicolumn{5}{c}{ all set to $(.25, .25, .25, .25)$} \\
InitialTemp & 10,000 & 100,000 & $10,000,000$ & 100,000 \\
TempFactor & 0.9999 & 0.99999 & 0.999999 & 0.99999 \\
\hline
\end{tabular}

lift opportunity in its middle. Such a disruption may add two unnecessary changeovers - to and from the single spreader - to the crane lift sequence.

Note that more computation time is allocated for attacking larger problems. Given this time limit, parameters InitialTemp and TempFactor are set so the SA procedure consists of three phases of roughly equal duration-(i) an initial exploration phase when almost any neighboring solution is accepted; (ii) a middle phase when the algorithm gradually transitions from being very accepting of neighbors to being very picky; and (iii) a final phase when virtually no inferior neighboring solutions are accepted. The increase (decrease) in parameters InitialTemp and TempFactor when going from problem size $3 \times 8$ to $10 \times 23(10 \times 23$ to $50 \times 50)$ follows from the fact that the allotted computation time grows at a faster (slower) rate than the problem size for these instances.

Table 3 shows the results for the first set of experiments that consider the 30 small problem instances of size $3 \times 8$. Each individual instance is specified by a code "TxSZnn" where $T$ is the number of tiers; $S$ is the number of stacks; "Z" takes the values ( $\mathrm{L}, \mathrm{M}, \mathrm{H})$ according to the container weight scenarios (light, medium, heavy); and "nn" denotes the instance number from 1 to 10. Each instance is considered in three ways - (A) using standard integer programming (IP) with no time limit ("CPLEX Alone"); (B) using the heuristic method with a $10 \mathrm{sec}$ time limit; and (C) using IP with no time limit where both the lower bound and the best solution found by the heuristic are passed to the solver at the outset ("CPLEX+LB+UB"). The heuristic method generates an average of eleven million neighboring solutions within the $10 \mathrm{sec}$ time limit. The best objective value (i.e. makespan) found by methods A, $\mathrm{B}$, and $\mathrm{C}$ are shown in columns $M_{\mathrm{CP}}, M_{H}$, and $M_{\mathrm{CP}+\mathrm{B}}$ respectively. Column $M_{H_{0}}$ shows the makespan of the initial feasible solution used in the heuristic method, and column LB shows the lower bound.

The results show that all three methods solve all instances to optimali- 
ty. Indeed, method A solves all instances to optimality within ten minutes; method B finds these optimal solutions within ten seconds; and method C solves all instances to optimality within three minutes. Importantly, the best solutions found by the heuristic (all of which happen to be optimal) are usually within $5 \%$ of the lower bound. Also, the objective value of the best heuristic solution (30.3) is about $7 \%$ better on average than that of the initial heuristic solution (32.6). Not surprisingly, the optimal values for the light instances are typically less than those for the medium instances. The same holds for the medium instances compared to the heavy instances.

Table 4 shows the results for the second set of experiments that consider the 30 medium-sized instances of size $5 \times 10$. Here, each instance is considered in three ways - (A) using IP with a one hour time limit ("CPLEX Alone"); (B) using the heuristic method with a 60 sec time limit; and (C) using IP with a one hour time limit where the lower bound and best solution found by the heuristic are passed to the solver at the outset ("CPLEX+LB+UB"). The heuristic method generates an average of 31 million neighboring solutions within the $60 \mathrm{sec}$ time limit. Column "Opt?" indicates if the associated solution is proven to be optimal or not.

The results for methods A and B show that the heuristic method performs better than IP. Indeed, in every instance, the best solution found by the heuristic in one minute is at least as good as the best solution found by CPLEX in an hour. Also, the average objective value of the best heuristic solution (62.6) is about $1.4 \%$ better than that for CPLEX (63.5). Note that the best solutions found by the heuristic (only two of which are known to be optimal) are about $6 \%$ higher on average than the lower bound. Also, the results for method $\mathrm{C}$ show that CPLEX is unable, in an hour, to improve upon the best heuristic solution provided to it at the outset for any instance. These observations give us confidence that the heuristic method is finding near-optimal or optimal solutions to these instances. Finally, we observe that the objective value of the best heuristic solution (62.6) is about $5 \%$ lower on average than that of the initial heuristic solution (65.7). Overall, the above results indicate that our IP framework is not a suitable solution method for instances with 50 or more containers. Thus, IP is not considered in the following experiments that consider larger problem instances.

Table 5 shows the results for the 60 largest problem instances - 30 large instances of size $10 \times 23$ and 30 very large instances of size $50 \times 50$. The results for the large (very large) instances are on the left (right). For each instance, we show the lower bound; the objective value of the best solution found by 
Table 3: Experimental results for instances of size $3 \times 8$. Optimal solutions are found by all three approaches.

\begin{tabular}{|c|c|c|c|c|c|c|c|c|c|}
\hline \multirow[b]{2}{*}{ Instance } & \multicolumn{2}{|c|}{ CPLEX Alone } & \multicolumn{3}{|c|}{ Heuristic (10 seconds) } & \multicolumn{2}{|c|}{ CPLEX+LB+UB } & \multirow[b]{2}{*}{ LB } & \multirow[b]{2}{*}{$\frac{M_{H}-L B}{M_{H}}$} \\
\hline & $M_{\mathrm{CP}}$ & Time (s) & $M_{H}$ & $M_{H_{0}}$ & $\frac{M_{H_{0}}-M_{H}}{M_{H}}$ & $M_{\mathrm{CP}+\mathrm{B}}$ & Time $(\mathrm{s})$ & & \\
\hline$\overline{3 \mathrm{x} 8 \mathrm{~L} 01}$ & 27.3 & 17 & 27.3 & 29.4 & $7.69 \%$ & 27.3 & 15 & 26.1 & $4.40 \%$ \\
\hline $3 \times 8 \mathrm{~L} 02$ & 26.1 & 12 & 26.1 & 31.8 & $21.84 \%$ & 26.1 & 0 & 26.1 & $0.00 \%$ \\
\hline x8L03 & 26.1 & 6 & 26.1 & 27.3 & $4.60 \%$ & 26.1 & 1 & 26.1 & $0.00 \%$ \\
\hline x8L04 & 29.4 & 211 & 29.4 & 31.8 & $8.16 \%$ & 29.4 & 112 & 27.3 & $7.14 \%$ \\
\hline ×8L05 & 21.6 & 1 & 21.6 & 28.2 & $30.56 \%$ & 21.6 & 1 & 21.6 & $0.00 \%$ \\
\hline $8 \mathrm{~L} 06$ & 27.3 & 80 & 27.3 & 33.9 & $24.18 \%$ & 27.3 & 71 & 26.1 & $4.40 \%$ \\
\hline $8 \mathrm{~L} 07$ & 24.9 & 4 & 24.9 & 28.2 & $13.25 \%$ & 24.9 & 0 & 24.9 & $0.00 \%$ \\
\hline $8 \mathrm{~L} 08$ & 30.6 & 573 & 30.6 & 31.8 & $3.92 \%$ & 30.6 & 157 & 28.5 & $6.86 \%$ \\
\hline 8L09 & 26.1 & 7 & 26.1 & 30.6 & $17.24 \%$ & 26.1 & 0 & 26.1 & $0.00 \%$ \\
\hline $3 \times 8 \mathrm{~L} 10$ & 28.2 & 424 & 28.2 & 31.5 & $11.70 \%$ & 28.2 & 75 & 26.1 & $7.45 \%$ \\
\hline Average & 26.8 & 133.5 & 26.8 & 30.5 & $14.31 \%$ & 26.8 & 43.2 & 25.9 & $3.02 \%$ \\
\hline 3x8M01 & 30.6 & 8 & 30.6 & 31.8 & $3.92 \%$ & 30.6 & 42 & 28.5 & $6.86 \%$ \\
\hline 3M02 & 30.9 & 37 & 30.9 & 30.9 & $0.00 \%$ & 30.9 & 25 & 29.7 & $3.88 \%$ \\
\hline M03 & 27.3 & 29 & 27.3 & 31.8 & $16.48 \%$ & 27.3 & 53 & 26.1 & $4.40 \%$ \\
\hline M04 & 34.2 & 76 & 34.2 & 35.7 & $4.39 \%$ & 34.2 & 55 & 32.1 & $6.14 \%$ \\
\hline M05 & 30.9 & 109 & 30.9 & 33.0 & $6.80 \%$ & 30.9 & 108 & 28.5 & $7.77 \%$ \\
\hline M06 & 27.3 & 7 & 27.3 & 33.0 & $20.88 \%$ & 27.3 & 0 & 27.3 & $0.00 \%$ \\
\hline M07 & 30.9 & 56 & 30.9 & 33.0 & $6.80 \%$ & 30.9 & 63 & 29.7 & $3.88 \%$ \\
\hline M08 & 32.1 & 17 & 32.1 & 33.0 & $2.80 \%$ & 32.1 & 24 & 30.9 & $3.74 \%$ \\
\hline M09 & 34.2 & 14 & 34.2 & 34.2 & $0.00 \%$ & 34.2 & 31 & 32.1 & $6.14 \%$ \\
\hline $3 \times 8 M 10$ & 28.5 & 6 & 28.5 & 30.6 & $7.37 \%$ & 28.5 & 33 & 27.3 & $4.21 \%$ \\
\hline Average & 30.7 & 35.9 & 30.7 & 32.7 & $6.94 \%$ & 30.7 & 43.4 & 29.2 & $4.70 \%$ \\
\hline 3x8H01 & 35.7 & 6 & 35.7 & 36.0 & $0.84 \%$ & 35.7 & 19 & 34.5 & $3.36 \%$ \\
\hline $3 \mathrm{x} 8 \mathrm{H} 02$ & 32.1 & 27 & 32.1 & 34.2 & $6.54 \%$ & 32.1 & 40 & 30.9 & $3.74 \%$ \\
\hline $3 \times 8 \mathrm{H} 03$ & 35.7 & 9 & 35.7 & 36.0 & $0.84 \%$ & 35.7 & 12 & 34.5 & $3.36 \%$ \\
\hline $3 \times 8 H 04$ & 29.7 & 10 & 29.7 & 31.8 & $7.07 \%$ & 29.7 & 0 & 29.7 & $0.00 \%$ \\
\hline 3x8H05 & 33.3 & 8 & 33.3 & 35.4 & $6.31 \%$ & 33.3 & 1 & 33.3 & $0.00 \%$ \\
\hline 3x8H06 & 34.5 & 7 & 34.5 & 35.4 & $2.61 \%$ & 34.5 & 19 & 33.3 & $3.48 \%$ \\
\hline $3 \times 8 \mathrm{H} 07$ & 32.1 & 24 & 32.1 & 32.1 & $0.00 \%$ & 32.1 & 38 & 30.9 & $3.74 \%$ \\
\hline $3 \times 8 \mathrm{H} 08$ & 32.1 & 8 & 32.1 & 35.4 & $10.28 \%$ & 32.1 & 16 & 30.9 & $3.74 \%$ \\
\hline $3 \times 8 \mathrm{H} 09$ & 34.2 & 22 & 34.2 & 34.2 & $0.00 \%$ & 34.2 & 25 & 32.1 & $6.14 \%$ \\
\hline 3x8H10 & 36.0 & 1 & 36.0 & 36.0 & $0.00 \%$ & 36.0 & 1 & 36.0 & $0.00 \%$ \\
\hline Average & 33.5 & 12.2 & 33.5 & 34.7 & $3.45 \%$ & 33.5 & 17.1 & 32.6 & $2.76 \%$ \\
\hline Ovrl Avg. & 30.3 & 60.5 & 30.3 & 32.6 & $8.24 \%$ & 30.3 & 34.6 & 29.2 & $3.49 \%$ \\
\hline
\end{tabular}


Table 4: Experimental results for instances of size $5 \times 10$.

\begin{tabular}{|c|c|c|c|c|c|c|c|c|c|c|c|}
\hline \multirow[b]{2}{*}{ Instance } & \multicolumn{3}{|c|}{ CPLEX Alone } & \multicolumn{3}{|c|}{ Heuristic (60 seconds) } & \multicolumn{3}{|c|}{ CPLEX+LB+UB } & \multirow[b]{2}{*}{$\mathrm{LB}$} & \multirow[b]{2}{*}{$\frac{M_{H}-L B}{M_{H}}$} \\
\hline & $\overline{M_{\mathrm{CP}}}$ & Time $(\mathrm{s})$ & Opt? & $\overline{M_{H}}$ & $M_{H_{0}}$ & $\frac{M_{H_{0}}-M_{H}}{M_{H}}$ & $M_{\mathrm{CP}+\mathrm{B}}$ & Time $(\mathrm{s})$ & Opt? & & \\
\hline 5x10L01 & 62.1 & 3861 & $?$ & 61.2 & 63.0 & $2.94 \%$ & 61.2 & 3605 & $?$ & 56.7 & $7.35 \%$ \\
\hline x10L02 & 55.2 & 3915 & $?$ & 55.2 & 63.6 & $15.22 \%$ & 55.2 & 3610 & $?$ & 53.1 & $3.80 \%$ \\
\hline x10L03 & 57.3 & 4001 & $?$ & 56.4 & 61.2 & $8.51 \%$ & 56.4 & 3608 & $?$ & 53.1 & $5.85 \%$ \\
\hline x10L04 & 56.1 & 3726 & $?$ & 55.2 & 58.8 & $6.52 \%$ & 55.2 & 3607 & $?$ & 51.9 & $5.98 \%$ \\
\hline x10L05 & 50.7 & 3673 & $?$ & 50.7 & 60.0 & $18.34 \%$ & 50.7 & 3603 & $?$ & 49.5 & $2.37 \%$ \\
\hline k10L06 & 66.0 & 3665 & $?$ & 63.3 & 67.8 & $7.11 \%$ & 63.3 & 3605 & $?$ & 57.9 & $8.53 \%$ \\
\hline L07 & 56.1 & 3850 & ? & 56.1 & 61.2 & $9.09 \%$ & 56.1 & 3604 & ? & 1.9 & $.49 \%$ \\
\hline 08 & 56.4 & 4114 & ? & 56.4 & 57.6 & $2.13 \%$ & 56.4 & 3605 & $?$ & 3.1 & $6.85 \%$ \\
\hline 09 & 51.6 & 3604 & $?$ & 51.6 & 58.8 & $13.95 \%$ & 1.6 & 3779 & $?$ & 9.5 & $4.07 \%$ \\
\hline x10L10 & 51.6 & 3600 & $?$ & 51.6 & 59.4 & $15.12 \%$ & 51.6 & 3663 & $?$ & 49.5 & $4.07 \%$ \\
\hline Avg. & 56.3 & 3800.9 & & 55.8 & 61.1 & $9.89 \%$ & 55.8 & 3628.9 & & 52.6 & $5.54 \%$ \\
\hline x10M01 & 7.8 & 3602 & $?$ & 64.8 & 66.6 & $2.78 \%$ & 64.8 & 3605 & $?$ & 60.3 & $6.94 \%$ \\
\hline 102 & 3.9 & 3766 & $?$ & 62.4 & 66.0 & $5.77 \%$ & 62.4 & 3604 & $?$ & 57.9 & $7.21 \%$ \\
\hline I03 & 3.9 & 3611 & $?$ & 63.6 & 66.0 & $3.77 \%$ & 63.6 & 3606 & $?$ & 60.3 & $5.19 \%$ \\
\hline I04 & 4.8 & 3769 & $?$ & 64.8 & 67.2 & $3.70 \%$ & 64.8 & 3606 & $?$ & 61.5 & $5.09 \%$ \\
\hline I05 & 6.0 & 3658 & $?$ & 64.8 & 64.8 & $0.00 \%$ & 4.8 & 3607 & $?$ & 61.5 & $5.09 \%$ \\
\hline & 62.1 & 3669 & $?$ & 57.6 & 59.7 & $3.65 \%$ & 57.6 & 3603 & $?$ & 54.3 & $5.73 \%$ \\
\hline & 0.6 & 3625 & $?$ & 59.7 & 63.0 & $53 \%$ & 9.7 & 3604 & $?$ & 4.3 & $9.05 \%$ \\
\hline & 68.1 & 3726 & ? & 66.9 & 69.9 & $4.48 \%$ & 6.9 & 3607 & $?$ & 61.5 & $8.07 \%$ \\
\hline & 4.2 & 3652 & ? & 62.1 & 63.3 & $1.93 \%$ & 2.1 & 3606 & $?$ & 57.9 & $6.76 \%$ \\
\hline 10 & 57.3 & 3602 & ? & 55.5 & 61.8 & $11.35 \%$ & 55.5 & 3604 & ? & 51.9 & $6.49 \%$ \\
\hline Avg. & 63.9 & 3668 & & 62.2 & 64.8 & $4.30 \%$ & 62.2 & 3605.2 & & 58.1 & $6.56 \%$ \\
\hline к10H01 & 74.4 & 223 & yes & 74.4 & 74.4 & $0.00 \%$ & 74.4 & 114 & yes & 72.3 & $2.82 \%$ \\
\hline 102 & 69.9 & 3641 & $?$ & 69.3 & 70.5 & $1.73 \%$ & 69.3 & 3608 & $?$ & 65.1 & $6.06 \%$ \\
\hline . $\mathrm{H} 03$ & 70.2 & 3606 & $?$ & 66.9 & 68.1 & $1.79 \%$ & 66.9 & 3603 & $?$ & 62.7 & $6.28 \%$ \\
\hline 810H04 & 69.3 & 3608 & $?$ & 66.9 & 67.8 & $1.35 \%$ & 66.9 & 3604 & $?$ & 61.5 & $8.07 \%$ \\
\hline x10H05 & 75.0 & 41 & yes & 75.0 & 75.0 & $0.00 \%$ & 75.0 & 104 & yes & 73.5 & $2.00 \%$ \\
\hline x10H06 & 72.9 & 3690 & $?$ & 72.9 & 73.2 & $0.41 \%$ & 72.9 & 3607 & $?$ & 66.3 & $9.05 \%$ \\
\hline x10H07 & 69.6 & 3602 & $?$ & 69.6 & 72.0 & $3.45 \%$ & 69.6 & 3608 & $?$ & 66.3 & $4.74 \%$ \\
\hline $5 \times 10 \mathrm{H} 08$ & 62.4 & 3669 & $?$ & 62.4 & 65.7 & $5.29 \%$ & 62.4 & 3623 & $?$ & 59.1 & $5.29 \%$ \\
\hline $5 \times 10 \mathrm{H} 09$ & 69.6 & 3609 & $?$ & 69.6 & 72.0 & $3.45 \%$ & 69.6 & 3606 & $?$ & 66.3 & $4.74 \%$ \\
\hline $5 \times 10 \mathrm{H} 10$ & 70.8 & 3616 & $?$ & 70.8 & 71.7 & $1.27 \%$ & 70.8 & 3606 & $?$ & 67.5 & $4.66 \%$ \\
\hline Avg. & 70.4 & 2930.5 & & 69.8 & 71.0 & $1.87 \%$ & 69.8 & 2908.3 & & 66.1 & $5.37 \%$ \\
\hline Ovrl Avg & 63.5 & 3466.5 & & 62.6 & 65.7 & $5.35 \%$ & 62.6 & 3380.8 & & 58.9 & $5.82 \%$ \\
\hline
\end{tabular}


the heuristic method within a $600 \mathrm{sec}$ time limit; and the objective value of the initial heuristic solution. The heuristic method generates an average of 39 (1.9) million neighboring solutions within the $600 \mathrm{sec}$ time limit for the instances of size $10 \times 23(50 \times 50)$.

The results show that the heuristic is finding near-optimal solutions to these instances. Indeed, the average objective value of the best heuristic solution for the instances of size $10 \times 23$ and $50 \times 50-275.2$ and 2936.8 respectively - is about $4 \%$ higher than the average lower bound - 263.8 and 2828.9 respectively - for these instances. Also, note that the quality of the heuristic solution improves as containers get heavier; on average, the makespan of the best heuristic solution is roughly $5 \%, 4 \%$, and $3 \%$ above the lower bound for the light, medium, and heavy instances respectively. This may be due to the fact that there are fewer opportunities for using the dual spreader - and therefore fewer choices - when containers are heavier. 
Table 5: Experimental results for instances of size $10 \times 23$ (left) and $50 \times 50$ (right) with the heuristic running for 600 seconds.

\begin{tabular}{|c|c|c|c|c|c|c|c|c|c|c|c|}
\hline istance & LB & $M_{H}$ & $M_{H_{0}}$ & $\frac{H_{0}-M_{H}}{M_{H}}$ & $\frac{M_{H}-L B}{M_{H}}$ & nstance & LB & $M_{H}$ & $M_{H_{0}}$ & $\begin{array}{l}\frac{H_{0}-M_{H}}{M_{H}} \\
\end{array}$ & $\frac{M_{H}-L B}{M_{H}}$ \\
\hline 0 & 8.3 & 37.9 & 249.6 & $4.92 \%$ & $4.04 \%$ & 0 & $\overline{96.9}$ & 28.6 & 6766 & 18307 & $501 \%$ \\
\hline & 5.9 & 4.6 & 4 & $\%$ & $3.71 \%$ & & & & & & $14 \%$ \\
\hline & .9 & 1.8 & 7 & $\%$ & 4.0 & & & & & & .78 \\
\hline & 8.3 & 9.4 & 5 & $\%$ & 4. & & .9 & .1 & .3 & $6 \%$ & 4.97 \\
\hline & .9 & 3.9 & 9 & & 4.9 & & & & & & .81 \\
\hline & .7 & 0.6 & 3 & $\%$ & & & & & & & $91 \%$ \\
\hline & & 7.5 & 4 & $\%$ & & & & & & & $.43 \%$ \\
\hline & & 3.0 & & $\%$ & & & & & & & $4.64 \%$ \\
\hline & & .7 & 7 & & & & & & & & $4.64 \%$ \\
\hline & 7.9 & 7.5 & 9.5 & $\%$ & 3.8 & & & & & & $5.44^{\circ}$ \\
\hline & 1.8 & 42.5 & 4.2 & $80 \%$ & $4.41 \%$ & & 92.9 & 23.5 & $\sigma x$ & $.74 \%$ & 4.98 \\
\hline & 1.3 & 8.1 & 1.4 & $\%$ & & & 92.1 & 02.5 & & & $3.80^{\circ}$ \\
\hline & 9.5 & 73.6 & 0.8 & & & & & & & & $78 \%$ \\
\hline & 3.1 & 74.8 & 3.2 & & & & & & & & $3.47 \%$ \\
\hline & 7 & 72.4 & 9.6 & & & & & & & & \\
\hline & & & & & & & & & & & וסט \\
\hline & & & & & & & & & & & $3.11 \%$ \\
\hline & & & & & & & & & & & \\
\hline & & & & & & & & & & & 4.047 \\
\hline & & & & & & & & & & & 4.129 \\
\hline & & 3.6 & 4.8 & & $4.28 \%$ & & & & & $.44 \%$ & 4.043 \\
\hline & 2.6 & 4.4 & $\overline{9.9}$ & & & & & 4.2 & 5.0 & $2 \%$ & 3.88 \\
\hline & & & & & & & & & & $9 \%$ & $2.60^{\circ}$ \\
\hline & & & & & & & & & & & $2.45 \%$ \\
\hline & & & & & & & & & & & $2.33^{\circ}$ \\
\hline & & & & & & & & & & & $2.79 \%$ \\
\hline & & & & & & & & & & $\%$ & $2.38^{\circ}$ \\
\hline & & & & & & & & & & $6 \%$ & $2.23 \%$ \\
\hline & & & & & & & & & & $5 \%$ & $2.32 \%$ \\
\hline & & & & & & & & & & $4 \%$ & $2.59 \%$ \\
\hline & & & & & & & & & 3277.8 & $8 \%$ & $2.33 \%$ \\
\hline & 9.1 & 9.6 & 5.3 & $1 \%$ & $3.39 \%$ & & & 3246.3 & 3256.2 & $.30 \%$ & $2.50 \%$ \\
\hline & .9 & 8.5 & 1.3 & $0.91 \%$ & $3.75 \%$ & & 2.5 & 272.8 & 3284.1 & $0.35 \%$ & $2.45 \%$ \\
\hline vrl Avg. & 263.8 & 275.2 & 281.8 & $2.57 \%$ & $4.16 \%$ & Ovrl Avg. & 2828.9 & 2936.8 & 2962.8 & $0.93 \%$ & 3.77 \\
\hline
\end{tabular}




\section{Conclusion}

In this paper, we proposed a new crane scheduling problem inspired by the dual-spreader quay crane, an emerging technology that has been deployed at some container ports around the world. The efficient operation of such cranes may allow containerships to be unloaded more quickly. We formulated the problem as a mixed-integer linear program, developed a tight lower bound on the optimal value, and devised a heuristic approach for handling large problem instances. The heuristic approach begins with an excellent initial feasible solution that effectively utilizes the problem structure. A simulated annealing framework was used to improve upon the initial feasible solution.

Numerical experiments indicate that the heuristic approach finds the same optimal solutions as CPLEX for small-sized instances. For mediumsized instances, the heuristic always outperforms CPLEX. The comparison between the optimal value and lower bound for small-sized instances suggests that the lower bound is tight, providing a good guide for solution quality. Overall, the heuristic approach produces crane schedules whose makespans, on average, are within $6 \%$ of the lower bound for each of the four problem sizes considered.

Although we solely consider the unloading of a storage bay, our approach can apply to the loading of a storage bay. Indeed, reversing the sequence of operations - single-spreader lifts, dual-spreader lifts, and changeoverscreates a schedule for loading a storage bay in the same amount of time in which it is unloaded.

Future work might proceed in several directions. First, the NP-hardness of this problem could be established. Second, other types of problem instances could be considered. In the instances we considered, parameters $H_{1}, H_{2}$, and $C$ were multiples of 0.3 , so the objective value was restricted to being a multiple of 0.3 . This might have hindered the search for better solutions within our optimization and heuristic approaches. Instances with more realistic positions of heavy and light containers - e.g. heavy containers at the bottom - could also be considered. Third, better optimization or heuristic approaches that use completely different search strategies could be developed. Finally, different variations of this problem - that include additional real-world constraints and/or consider the distance moved by the spreader - might be considered. 


\section{Acknowledgements}

No ghost writers or ghost computer programmers were involved in the production of this manuscript. The authors thank three anonymous reviewers for several helpful comments that strengthened this paper.

\section{References}

Angeloudis, P., Bell, M. G., 2011. A review of container terminal simulation models. Maritime Policy \& Management 38 (5), 523-540.

Bierwirth, C., Meisel, F., 2010. A survey of berth allocation and quay crane scheduling problems in container terminals. European Journal of Operational Research 202 (3), 615-627.

Bierwirth, C., Meisel, F., 2015. A follow-up survey of berth allocation and quay crane scheduling problems in container terminals. European Journal of Operational Research 244 (3), 675-689.

Carlo, H. J., Vis, I. F., Roodbergen, K. J., 2014a. Storage yard operations in container terminals: Literature overview, trends, and research directions. European Journal of Operational Research 235 (2), 412-430.

Carlo, H. J., Vis, I. F., Roodbergen, K. J., 2014b. Transport operations in container terminals: Literature overview, trends, research directions and classification scheme. European Journal of Operational Research 236 (1), $1-13$.

Carlo, H. J., Vis, I. F., Roodbergen, K. J., 2015. Seaside operations in container terminals: Literature overview, trends, and research directions. Flexible Services and Manufacturing Journal 27 (2-3), 224-262.

Chao, S.-L., Lin, Y.-J., 2011. Evaluating advanced quay cranes in container terminals. Transportation Research Part E: Logistics and Transportation Review 47 (4), 432-445.

Chen, J. H., Lee, D.-H., Cao, J. X., 2011. Heuristics for quay crane scheduling at indented berth. Transportation Research Part E: Logistics and Transportation Review 47 (6), 1005-1020. 
Chen, L. H., Cao, J. X., Zhao, Q. Y., 2014. Tandem lift quay cranes and yard trucks scheduling problem at container terminals. In: Applied Mechanics and Materials. Vol. 505. Trans Tech Publ, pp. 927-930.

Cheung, R. K., Li, C.-L., Lin, W., 2002. Interblock crane deployment in container terminals. Transportation Science 36 (1), 79-93.

Choi, S.-H., Im, H., Lee, C., 2014. Development of an operating system for optimization of the container terminal by using the tandem-lift quay crane. In: Park, J. J., Stojmenovic, I., Choi, M., Xhafa, F. (Eds.), Future Information Technology. Springer, Berlin, pp. 399-404.

Dohn, A., Clausen, J., 2010. Optimising the slab yard planning and crane scheduling problem using a two-stage heuristic. International Journal of Production Research 48 (15), 4585-4608.

Gharehgozli, A. H., Laporte, G., Yu, Y., de Koster, R., 2015a. Scheduling twin yard cranes in a container block. Transportation Science 49 (3), 686705.

Gharehgozli, A. H., Roy, D., de Koster, R., 2015b. Sea container terminals: New technologies and or models. Maritime Economics \& Logistics.

Goussiatiner, A., 2007a. In pursuit of productivity. Container Management August.

Goussiatiner, A., 2007b. In pursuit of productivity 2. Container Management September.

Imai, A., Chen, H. C., Nishimura, E., Papadimitriou, S., 2008. The simultaneous berth and quay crane allocation problem. Transportation Research Part E: Logistics and Transportation Review 44 (5), 900-920.

Iris, Ç., Pacino, D., Ropke, S., Larsen, A., 2015. Integrated berth allocation and quay crane assignment problem: Set partitioning models and computational results. Transportation Research Part E: Logistics and Transportation Review 81, 75-97.

Kim, K. H., Kim, K. Y., 1999. An optimal routing algorithm for a transfer crane in port container terminals. Transportation Science 33 (1), 17-33. 
Kim, K. H., Park, Y.-M., 2004. A crane scheduling method for port container terminals. European Journal of Operational Research 156 (3), 752-768.

Kung, Y., Kobayashi, Y., Higashi, T., Sugi, M., Ota, J., 2014. Order scheduling of multiple stacker cranes on common rails in an automated storage/retrieval system. International Journal of Production Research 52 (4), 1171-1187.

Lee, C.-Y., Liu, M., Chu, C., 2015. Optimal algorithm for the general quay crane double-cycling problem. Transportation Science 49 (4), 957-967.

Lee, D.-H., Chen, J. H., Cao, J. X., 2011. Quay crane scheduling for an indented berth. Engineering Optimization 43 (9), 985-998.

Li, F., Sheu, J.-B., Gao, Z.-Y., 2015. Solving the continuous berth allocation and specific quay crane assignment problems with quay crane coverage range. Transportation Science 49 (4), 968-989.

Li, W., Goh, M., Wu, Y., Petering, M. E. H., de Souza, R., Wu, Y. C., 2012. A continuous time model for multiple yard crane scheduling with last minute job arrivals. International Journal of Production Economics $136(2), 332-343$.

McCarthy, P. W., Jordan, M. A., Wright, L., 2007. Dual-hoist, tandem 40 crane considerations. Port Technology International 34, 111-113.

Meisel, F., Bierwirth, C., 2013. A framework for integrated berth allocation and crane operations planning in seaport container terminals. Transportation Science 47 (2), 131-147.

Moccia, L., Cordeau, J.-F., Gaudioso, M., Laporte, G., 2006. A branchand-cut algorithm for the quay crane scheduling problem in a container terminal. Naval Research Logistics (NRL) 53 (1), 45-59.

Ng, W., Mak, K., 2006. Quay crane scheduling in container terminals. Engineering Optimization 38 (6), 723-737.

Peterson, B., Harjunkoski, I., Hoda, S., Hooker, J. N., 2014. Scheduling multiple factory cranes on a common track. Computers \& Operations Research 48, 102-112. 
Song, J.-H., 2011. Tandem operation and double cycling in container terminals. Port Technology International 51, 73-79.

Stahlbock, R., Voß, S., 2008. Operations research at container terminals: a literature update. OR Spectrum 30 (1), 1-52.

Steenken, D., Voß, S., Stahlbock, R., 2004. Container terminal operation and operations research-a classification and literature review. OR spectrum 26 (1), 3-49.

Tang, L., Zhao, J., Liu, J., 2014. Modeling and solution of the joint quay crane and truck scheduling problem. European Journal of Operational Research 236 (3), 978-990.

Türkoğulları, Y. B., Taşkın, Z. C., Aras, N., Altınel, İ. K., 2014. Optimal berth allocation and time-invariant quay crane assignment in container terminals. European Journal of Operational Research 235 (1), 88-101.

Unsal, O., Oguz, C., 2013. Constraint programming approach to quay crane scheduling problem. Transportation Research Part E: Logistics and Transportation Review 59, 108-122.

Vacca, I., Salani, M., Bierlaire, M., 2013. An exact algorithm for the integrated planning of berth allocation and quay crane assignment. Transportation Science 47 (2), 148-161.

Vis, I. F., Carlo, H. J., 2010. Sequencing two cooperating automated stacking cranes in a container terminal. Transportation Science 44 (2), 169-182.

Vis, I. F., De Koster, R., 2003. Transshipment of containers at a container terminal: An overview. European journal of operational research 147 (1), $1-16$.

World Cargo News, 2007. Make mine a double - or even a triple.

Wu, Y., Li, W.-K., Petering, M., Goh, M., de Souza, R., 2015. Scheduling multiple yard cranes with crane interference and safety distance requirement. Transportation Science 49 (4), 990-1005.

Xing, Y., Yin, K., Quadrifoglio, L., Wang, B., 2012. Dispatch problem of automated guided vehicles for serving tandem lift quay crane. Transportation Research Record (2273), 79-86. 